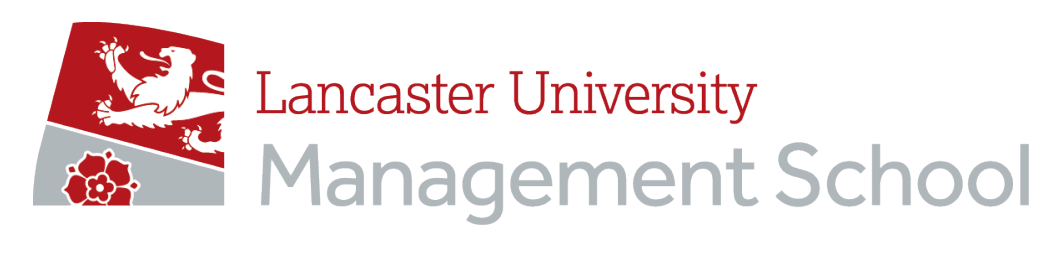

\author{
Economics Working Paper Series
}

2019/015

\title{
Technological change, campaign spending and Polarization
}

Pau Balart, Agustin Casas and Orestis Troumpounis

The Department of Economics

Lancaster University Management School

Lancaster LA1 4YX

UK

(C) Authors

All rights reserved. Short sections of text, not to exceed two paragraphs, may be quoted without explicit permission, provided that full acknowledgement is given. 


\title{
Technological change, campaign spending and polarization*
}

\author{
Pau Balart ${ }^{\dagger} \quad$ Agustin Casas $^{\ddagger} \quad$ Orestis Troumpounis ${ }^{\S}$
}

December 12, 2018

\begin{abstract}
We focus on changes in technology and campaign management to study the documented simultaneous increase in campaign spending and polarization. In our model, some voters are ideological while others are impressionable. If the distribution of voters between types is endogenous and depends on parties' platform choices, our results show that a) an increase in the effectiveness of electoral advertising or a decrease in the electorate's political awareness, surely increases polarization and may also increase campaign spending, while b) a decrease in the cost of advertising does not affect neither polarization nor spending.
\end{abstract}

Keywords: electoral competition, campaign spending, impressionable voters, semiorder lexicographic preferences

JEL codes: D 72 .

*We appreciate valuable feedback from participants in the following events: 2017 Contests: Theory and Evidence Conference, 2018 European Winter Meeting of the Econometric Society, Petralia Sottana 2018: Applied Economics Workshop, 2018 Political Economy Workshop Rotterdam, 2018 Madrid Political Economy Workshop, 3d Grode Workshop: Game Theory. Financial support from the Agencia Estatal de Investigacion (AEI) and the European Regional Development Funds (ERDF) through grants ECO2017-86305-C4-1-R (Balart) and ECO2017-85763-R (Casas) is acknowledged. Balart also acknowledges financial support by the Fundación Ramón Areces.

${ }^{\dagger}$ Department of Business Economics, Universitat de les Illes Balears, Carretera de Valldemossa, km 7.5 (Edifici Jovellanos), 07022 Palma de Mallorca, Spain. pau.balart@uib.cat

${ }^{\ddagger}$ CUNEF, C/Leonardo Prieto Castro, 2. Madrid, 28040, Spain. acasas@u.northwestern.edu

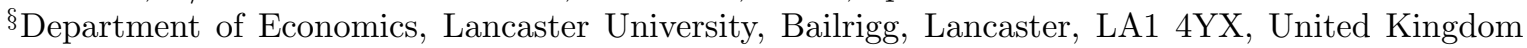
and Department of Economics, University of Padova, via del Santo 33, 35123 Padova, Italy. troumpounis@gmail.com 


\section{Introduction}

A well documented fact in US politics is the simultaneous increase of campaign spending and polarization. Updated measures indicate that polarization in 2015 was at the highest level since the era of Reconstruction, with this trend being well documented since earlier work by Poole and Rosenthal (1984) ! Also campaign spending, with the exception of the 2016 presidential election, has been steadily increasing since 1960 with a noteworthy increase in the 2008 and 2012 presidential elections.2 In this paper, we provide a new theoretical explanation for the occurrence of these phenomena by linking campaign spending and polarization with recent technological advances and changes in the management of electoral campaigns.

Focusing on technology is relevant because while it is still gaining importance (e.g., Obama's campaigns, the use of big data, and social media (Nickerson and Rogers, 2014)), research so far has not drawn conclusive evidence on its effects on electoral competition (Herrera, Levine, and Martinelli, 2008). Dating back to the introduction of nationwide TV in the 1960s party centered non-professional campaigns were gradually abandoned. Instead parties had to rely on professional campaign management due to the emergence of multiple channels and 24/7 news coverage and the internet revolution in the mid-1990s (Norris, 2000; Sabato, 1981). During recent years, the importance of technology is at its peak and it is widely acknowledged that campaigns involve highly sophisticated tools. The main question we aim to address is: What is the effect of such changes on electoral competition?

In line with changes in technology and campaign management, we present a theory where technology affects a) the effectiveness of electoral advertising -e.g., due to the professionalization of campaign management and advanced targeting technologies, b) the costs of electoral advertising - e.g., due to the possibility of reaching larger masses at a low marginal cost, and c) the electorate's political awareness -e.g., due to the presence of various sources of information. To sum up our main results, an increase in the effectiveness of electoral advertising or a decrease in the electorate's awareness increase polarization and may also increase campaign spending. On the contrary, changes in the marginal cost of advertising cannot explain changes neither in polarization nor campaign spending.

We analyze the effects of technological changes on campaign spending and polarization by combining the two seminal models of Downs (1957) and Tullock (1980). In particular, we assume that two office motivated parties first choose their electoral platforms and then decide upon the optimal level of costly electoral advertising. Voters can be either

\footnotetext{
${ }^{1}$ See http://pooleandrosenthal.com/political_polarization_2015.htm

${ }^{2}$ See https://www.fec.gov/data/elections/president/2016/.
} 
impressionable or ideological as in the seminal papers by Baron (1994); Grossman and Helpman (1996). As common in this literature, ideological voters support the party that proposes the platform closest to their bliss point. Hence, parties compete for a share of ideological voters as if they were competing in a Downsian model of electoral competition. On the contrary, impressionable voters are swayed towards one party or the other through costly electoral advertising. Given each party's electoral advertising, the effectiveness of the latter determines the fraction of impressionable voters that supports each of them. Differing from previous literature, we endogenize the division of voters across impressionable and ideological. Such division depends on the differentiation between the proposed platforms, with the fraction of ideological voters increasing in polarization. This assumption captures the idea that the more diverse platforms are, more voters vote based on their ideological preferences since platforms become "salient". On the contrary, when parties' platforms are similar, voters may have a "hard" time or little interest in distinguishing them, and turn to electoral advertising that determines (probabilistically) their voting behavior. Alas intuitive, one way to formalize this behavior is that voters' preferences are described by a lexicographic semiorder (see Luce 1956, Tversky 1969; Rubinstein 1988; Leland 1994; Manzini and Mariotti 2012), consistent with the notion of the "just noticeable difference" 3 With such preferences, each individual chooses which party to support on the basis of platforms, but only if those are different "enough" (i.e., above a certain threshold). If the platforms are not sufficiently different, then the voter is influenced exclusively by parties' electoral advertising.

The above sketched model encompasses the effect of technological changes on electoral competition through three distinct and non-mutually exclusive channels. The first two reflect the way campaigns for impressionable - Tullock- voters are conducted and how technology and changes in campaign management affect a) the effectiveness, and b) the cost of electoral advertising. One could reasonably argue that recent technological advances have increased the effectiveness of electoral advertising since campaigns can be well targeted, and have decreased the marginal cost of advertising given the possibility of reaching large masses. The third channel captures how technology affects electoral competition through the electorate's political awareness and the endogenous division of voters to impressionable and ideological. More precisely we focus on the conversion rate at which impressionable voters become ideological as polarization increases. Although not straightforward how this the conversion rate has evolved, we show how it affects electoral competition.

Our setup proves relatively tractable. In contrast to previous contributions with en-

\footnotetext{
${ }^{3}$ In experimental psychology, the Weber-Fechner law remarks that the "just noticeable difference" is not necessarily influenced by the physiological but rather by psychological factors. The law states that the just-noticeable difference is increasing in the absolute level of the subtracts (Falmagne, 2002).
} 
dogenous platforms and electoral advertising - that we later discuss, our model: i) has a unique Nash equilibrium in pure strategies when parties are symmetric, and ii) can be solved allowing heterogeneity in parties' marginal cost of campaigning. But most importantly, in equilibrium, several comparative statics arise regarding the effect of technological advances on electoral competition.

Consider first an increase in the effectiveness of electoral advertising. Since every dollar spent on campaigns leads to higher returns, parties have incentives to symmetrically increase their campaign spending (spending effect). To mitigate such increase in electoral advertising, parties have incentives to polarize their platforms and reduce the number of impressionable voters and hence their spending (polarization effect). If the spending effect dominates, campaign spending and polarization increase simultaneously and can explain the observed trends in US politics. If the polarization effect dominates, an increase in the electoral effectiveness is overcompensated by an increase in polarization and campaign spending decreases. Actually, the effectiveness of electoral advertising proves crucial if one wants to explain the simultaneous increase in campaign spending and polarization in terms of campaign management. A decrease in the marginal costs of running a campaign does not affect neither polarization nor total spending. While a decrease in the marginal cost of advertising leads to more advertising, the lower marginal cost of the latter leaves campaign spending and polarization unaffected. Finally, the conversion rate at which impressionable voters become ideological when polarization increases affects both campaign spending and polarization in a similar manner as a change in the effectiveness. If one wants to explain the simultaneous increase in polarization and spending exclusively through this channel, then the conversion rate has been decreasing, meaning that voters do not respond much to changes in platforms for example due "media malaise", possibly associated with mistrust of politicians and disenchantment with politics (Norris, 2000; Newton, 1999).

\subsection{Related Literature}

In terms of results, our work complements existing models of platform choice with endogenous advertising. The closest paper in terms of research question and methodology is by Herrera et al. (2008) who explicitly model changes in the targeting effectiveness and its effect on polarization and spending. Contrary to us, they show that an improved campaign technology reduces polarization. This is becasue while in our model polarization softens competition in the electoral advertising stage (as the endogenous valence literature), in their model it has the exact opposite effect. Prummer (2018) focuses on changes in targeting technology and fragmentation of media networks as determinants of polarization. Moving away from a targeting story, Rivas (2017) provides an alternative 
justification for the simultaneous increase in polarization and campaign spending in a model where the latter is financed through lobbies.

The structure of our model, where parties first choose platforms and then spending, resembles existing models of endogenous valence (e.g., Ashworth and Bueno de Mesquita (2009); Zakharov (2009); Carrillo and Castanheira (2008); Iaryczower and Mattozzi (2013) among others). In that literature, voters typically have additive separable preferences over platforms and valence (i.e., electoral advertising). In the closest work to ours Ashworth and Bueno de Mesquita, 2009; Zakharov, 2009), platform diversification softens the competition in the valence accumulation stage. 4 These dynamics are exactly the ones presented in our model through the endogenous division of voters to ideological and impressionable. Our model, however, proves more tractable and permits the analysis of the effects of technological changes on electoral competition. In contrast to Ashworth and Bueno de Mesquita (2009), our model under symmetry admits a pure strategy equilibrium in the campaign stage setting and does not require that voters' ideologies are uniformly distributed. A non-uniform distribution is also permitted in Zakharov (2009) but only when focusing on local Nash equilibria. In contrast, we are able to characterize Nash equilibria in pure strategies for a general distribution of voters' ideology (symmetric and log-concave in this paper) and perform relevant comparative statics. Also in contrast to the previous models, we are also able to solve the model and obtain results when parties have heterogeneous campaign costs, for example due to an incumbency advantage Meirowitz, 2008; Pastine and Pastine, 2012).

Summing up, one could think of our model as one of endogenous valence where rather than additive separable preferences over platforms and campaigns, individuals have semiorder lexicographic preferences (see Luce 1956; Tversky 1969: Manzini and Mariotti 2012; Rubinstein 1988; Leland 1994) and is in line with salience models where decision makers overweight attributes that exhibit greater differences in the available choice set (Bordalo, Gennaioli, and Shleifer 2012, 2013a,b, 2015; Bushong, Rabin, and Schwartzstein 2017; Köszegi and Szeidl 2012; Spiegler 2014). Semiorder lexicographic preferences can be seen as a particular case of salience in which: a) only the difference in one attribute (policy platforms) is relevant to assign the weight of each dimension, and b) individual weights on each attribute are discrete and take value 0 or 1 . In aggregate terms, our assumption on the endogenous division of voters across types has a similar effect to the one of salience at the individual level: the smaller the distance between platforms, the smaller is their weight on the final outcome of the electoral competition. Callander and Wilson (2006) and Nunnari and Zápal (2017) introduce related context-dependent preferences in

${ }^{4}$ The opposite effect may occur when parties have ideological motives (Epstein and Nitzan, 2004 Cardona, De Freitas, and Rubí-Barceló, 2018) or there is uncertainty regarding the valence investment (Carrillo and Castanheira, 2008). 
political economy models. In the former, the utility of voting depends not only on the direct benefits of turnout but also on the context, i.e., the candidates' polarization. In the latter, the authors provide a model of "focusing", where voters attention is captured more on the issues that candidates' proposals differ more. Similarly, Amorós and Puy (2013); Aragonés, Castanheira, and Giani (2015); Denter (2017) focus on electoral competition models when parties have the ability to affect the relative salience of different issues via their strategic actions (e.g., allocation of time or effort). In our model, parties' strategic actions affect the salience of platforms versus advertising, and hence voters in the society split among those casting votes either in an informed or uninformed manner. Therefore, although very different in nature, our model links with recent literature where some voters may be partially informed regarding parties' policy proposals Aragonès and Xefteris, 2017; Eguia and Nicolò, 2018).

Finally, our model contributes to the contest theory literature (see Corchón (2007); Konrad (2009); Serena and Corchón (2018) for surveys) since parties compete for a share of impressionable voters as if they were competing in a Tullock contest (with the "noise" of the latter capturing the effectiveness of electoral advertising). Notice that the value of the "prize" (of the contest) allocated based on electoral advertising is endogenous and depends on platform selection. Consequently, by fixing closer platforms parties not only attract more voters from their competitor as in any Downsian model, but also increase the share of Tullock voters for which rent-dissipation arises (Tullock, 1980; Nitzan, 1994). In terms of results, the value of the prize depending on platforms provides a result in contrast to most of the contest literature where the relationship of electoral advertising and the noise of the contest success is monotonic.

\section{Model}

Let two political parties $i \in\{L, R\}$ first propose (and commit to) platforms $x_{i}$ in the policy space $X=[0,1]$ and then choose the level of campaign advertising $e_{i} \geq 0$. Without loss of generality, we assume $x_{L} \leq x_{R}$. Let $S_{i}\left(x_{L}, x_{R}, e_{L}, e_{R}\right)$ be the vote share for party $i$ and $c_{i}\left(e_{i}\right)=\mu_{i} e_{i}$ the cost of advertising, with $\mu_{i}>0$ denoting the constant marginal cost of advertising. Without loss of generality, we assume $\mu_{L} \leq \mu_{R}$. Parties' are office motivated with payoffs $\Pi_{i}=S_{i}\left(x_{L}, x_{R}, e_{L}, e_{R}\right)-c_{i}\left(e_{i}\right) !^{5}$

Voters have a preferred policy $x$ drawn from distribution $G(x)$ with corresponding density $g(x)$ symmetric and log-concave (i.e., $(\ln g(x))^{\prime \prime} \leq 0$ ), with full support in X

\footnotetext{
${ }^{5}$ Here each party's objective is to maximize its vote share net of the campaign costs. Alternatively, $S_{i}\left(x_{L}, x_{R}, e_{L}, e_{R}\right)$ can also be interpreted as the probability of winning by assuming parties' uncertainty on voters' ideology as in Aragonès and Xefteris (2017).

${ }^{6}$ Under symmetric campaign costs, our results can be extended to asymmetric distributions of ideol-
} 
Independent of their ideal policy some voters are ideological and some are impressionable. The ideological citizens vote sincerely for the party whose proposed platform is closer to them and split their vote if the proposed platforms coincide ( $\grave{a}$ la Downs). The utility of a voter with ideology $x$ that votes for party $i$ is $\left.u_{x}(i)=-\left|x-x_{i}\right|\right]^{7}$

The impressionable citizens' vote depends only on electoral advertising. In particular, we assume that given parties' advertising, the probability that an impressionable citizen votes for party $i$ is determined $\grave{a}$ la Tullock and hence equal to $e_{i}^{\eta} /\left(e_{L}^{\eta}+e_{R}^{\eta}\right)$, where parameter $\eta>0$ captures the effectiveness of electoral advertising 8 If $\eta \rightarrow 0$, impressionable voters split equally across the two parties. However, as $\eta$ increases, the allocation of impressionable voters across parties becomes more responsive to electoral advertising. Impressionable voters voting on the basis of persuasive electoral advertising is a standard assumption in this literature (see for example the seminal papers by Baron (1994); Grossman and Helpman (1996) and a large literature thereafter). The specific proposed function is the seminal contest success function (CSF) introduced by Tullock (1980). This function is extensively used in the literature and apart from tractability also satisfies relevant axiomatic properties (Skaperdas, 1996) and can be micro-founded in a reasonable manner (also in our setting, see Section 5.4 of the Appendix) $!^{9}$

The endogenous division of voters across ideological and impressionable depends on the level of polarization. Let $y=x_{R}-x_{L} \in X$ be the platforms' polarization and $F(y)$ a continuous cumulative distribution function, log-concave (i.e., $\left.(\ln F(y))^{\prime \prime} \leq 0\right)$, with corresponding density $f(y)$ with full support in $\mathrm{X}$. The share of ideological voters is $F(y)$, and therefore the share of impressionable voters is $1-F(y)$.

It is informative to briefly stress how the above presentation of our model in aggregate terms reflects voters' individual behavior. Consider voters with semiorder lexicographic preferences. Let the first attribute reflect policies and the second attribute electoral advertising. Each voter draws a preferred policy $x$ from $G(x)$ and a level of "sensitivity" $\phi$ from $F_{\phi}(y)=\operatorname{Pr}(\phi \leq y)$, denoted $F(y)$ for simplicity. The value of $\phi$ determines if the voter is voting based on policies or advertising. If polarization is greater than $\phi$, the voter is ideological. If polarization is less than $\phi$ the voter is impressionable. That is, as platforms diverge, an individual voter is ideological with ex-ante probability $F(y)$,

ogy (available upon request).

${ }^{7}$ The assumption of a particular distance function is made without loss of generality.

${ }^{8}$ We assume $\eta>0$ to focus on cases in which our model differs from a Downsian model. If $\eta=0$, equilibrium spending is always zero and platform choice coincides with that of the standard Downsian model.

${ }^{9}$ The campaign stage for impressionable voters is resolved via Tullock's ratio-form CSF that facilitates the comparative statics of our model. In the symmetric case, our results would have the same qualitative features if we considered the difference-form CSF proposed by Alcalde and Dahm (2007), the tractable noise CSF proposed by Amegashie (2006) or the relative-difference CSF by Beviá and Corchón (2015) under the parameter restrictions proposed by Balart, Chowdhury, and Troumpounis (2017). 
and impressionable with ex-ante probability $1-F(y)$. Hence, despite the stark partition of voters into ideological and impressionable presented in the main text, an individual voter's expected behavior a priori depends both on platforms and advertising. To avoid additional notation we focus on the aggregate version of the model throughout the paper. The microfoundations of individuals' behavior (in terms of semiorder lexicographic preferences as above and in terms of a salience model) and the effect of advertising on individual voting behavior (and the rise of the Tullock CSF) are detailed in Section 5.4 of the Appendix.

The timing of the game is as follows: At $t=1$, the political parties simultaneously choose the political platforms that maximizes their payoff. At $t=2$, having observed the platforms choices and the share of impressionable voters determined by the polarization, parties choose the advertising levels. Finally, at $t=3$, voters vote. Given the nature of our game, we focus on subgame perfect Nash equilibria in pure strategies.

\section{Results}

Given the described game, let $\bar{x}=\frac{x_{L}+x_{R}}{2}$ be the indifferent ideological voter for $x_{L} \neq x_{R}$. Ideological voters with $x \leq \bar{x}$ vote for $L$, while the remaining ones vote for $R$. Thus, party $L$ obtains a share $S_{I d l}^{L}=G(\bar{x})$ of the ideological voters and party $R, S_{I d l}^{R}=1-$ $G(\bar{x})$. If $x_{L}=x_{R}$, then $S_{I d l}^{R}=S_{I d l}^{L}=\frac{1}{2}$. Given that the individual probability that an impressionable citizen votes for party $i$ is $\frac{e_{i}^{\eta}}{e_{L}^{\eta}+e_{R}^{\eta}}$, the expected share of impressionable votes to party $i$ is $S_{I m p}^{i}=\frac{e_{i}^{\eta}}{e_{L}^{\eta}+e_{R}^{\eta}}$ for party $i$. Hence, the expected vote share obtained by the parties can be then written as a weighted average of the previous two:

$$
S_{i}\left(x_{L}, x_{R}, e_{L}, e_{R}\right)=F(y) S_{I d l}^{i}\left(x_{L}, x_{R}\right)+(1-F(y)) S_{I m p}^{i}\left(e_{L}, e_{R}\right) .
$$

This expression highlights the effect of platform choices in our game. First, platform choice affects how ideological voters split between the parties (via $S_{I d l}^{i}\left(x_{L}, x_{R}\right)$ ). Second, platform choice affects the ideological-impressionable composition of the electorate (via $F(y))$. As common in Downsian type models, converging towards the opponent is beneficial due to the relocation of the indifferent voter. However, in our model platform convergence results into an increase in the share of impressionable voters, and hence a tougher competition in the (costly) advertising stage. The above trade off makes platforms' choice a non-trivial task. 


\subsection{Symmetric parties}

For illustrative purposes, and to highlight our main results in the simplest framework, we first pay attention to parties having identical marginal costs, i.e., $\mu_{A}=\mu_{B}=\mu$. Recall that voters' behavior is essentially parametric and hence the last stage in our backward induction reasoning is the choice of advertising. Equilibrium advertising can be solved as effort in a Tullock contest with symmetric players, in which the prize of winning equals the share of impressionable voters. The equilibrium in this stage is described in the lemma below

Lemma 1. For all $\eta \leq 2$ there exists a unique Nash equilibrium in the campaign stage and advertising is given by $e_{i}^{*}\left(x_{L}, x_{R}\right)=\left(1-F\left(x_{R}-x_{L}\right)\right) \frac{\eta}{4 \mu}$, for all $i$.

\section{All proofs appear in the Appendix.}

Our first Lemma draws from previous results in the contest theory literature. It characterizes the equilibrium advertising levels while stating a condition on the campaigns' effectiveness $\eta$ such that an equilibrium in pure strategies exists. If the campaigns are not effective "enough" (i.e., $\eta \leq 2$ ), an equilibrium in pure strategies exists and is unique with advertising being: a) increasing in the campaign effectiveness $\eta$, b) decreasing in the marginal cost $\mu$, and c) decreasing in the platforms' polarization $y$ (recall that $\left.y=x_{R}-x_{L}\right) 10$ Note that the symmetric spending in equilibrium implies that in equilibrium impressionable voters split between the two parties (i.e., $S_{I m p}^{L}=S_{I m p}^{R}=0.5$ ).

Anticipating the advertising levels in the second stage, the political parties' maximization problem in the first stage is to choose the platform that maximizes their payoff. For instance, for party $L$, the payoff at $t=1$ is $\Pi_{L}\left(x_{L}, x_{R}, e_{L}^{*}\left(x_{L}, x_{R}\right), e_{R}^{*}\left(x_{L}, x_{R}\right)\right)$, which can be written as:

$S_{L}\left(x_{L}, x_{R}\right)-c_{L}\left(e_{L}^{*}\left(x_{L}, x_{R}\right)\right)=F\left(x_{R}-x_{L}\right) S_{I d l}^{L}\left(x_{L}, x_{R}\right)+\left(1-F\left(x_{R}-x_{L}\right)\right) S_{I m p}^{L}-\mu e_{L}^{*}\left(x_{L}, x_{R}\right)$

where $S_{\text {Imp }}^{L}=\frac{1}{2}$ and $S_{I d l}^{L}=G(\bar{x})$ if $x_{L} \neq x_{R}$ or $S_{\text {Idl }}^{L}=\frac{1}{2}$ if $x_{L}=x_{R}$.

The trade-off parties face is now evident. Consider that for a given set of platforms $\left(x_{L}, x_{R}\right)$, the leftist party chooses to propose a less extreme platform. On the one hand, the indifferent voter is more to the right, which has a positive effect on $S_{I d l}^{L}$ as in a standard Downsian model. On the other hand, it converts some ideological voters to impressionable ones, which by Lemma1 1 increases the spending on advertising in the second stage of game.

\footnotetext{
${ }^{10}$ If campaigns are effective "enough" (i.e., $\eta>2$ ), there is no equilibrium in pure strategies in that stage and all mixed-strategy equilibria are payoff equivalent (Alcalde and Dahm, 2010) with parties' expected payoffs in that stage zero and $\left.E\left(e_{i}^{*}\left(x_{L}, x_{R}\right)\right)=\left(1-F\left(x_{R}-x_{L}\right)\right) \frac{1}{2 \mu}\right)$. For recent advances on the properties of such mixed equilibria and relevant literature refer to Ewerhart (2015).
} 
Similar to Tirole (1988) and Ashworth and Bueno de Mesquita (2009) divergence is a tool of softening competition in the vertical dimension (the advertising stage in our model).

The importance of each of the two forces present in the trade-off is determined by the rate at which impressionable voters become ideological as polarization increases (i.e., $\left.\frac{f(y)}{F(y)}\right)$ and the mass of voters around the indifferent voter $g(\bar{x})$. The relative importance of these two forces is moderated by the campaign effectiveness $\eta$. We characterize the equilibrium of the platform stage in the following proposition.

Proposition 1. Let $\eta \leq 2$ and $\bar{y}$ implicitly defined by $\frac{f(\bar{y})}{F(\bar{y})}=\frac{2}{\eta} g\left(\frac{1}{2}\right)$. For any $\mu>0$ there exists a unique subgame perfect Nash equilibrium.

For $F(0)>0$, the following equilibrium types arise:

- (Convergent equilibrium) $x_{L}^{*}=x_{R}^{*}=\frac{1}{2}$, if $\frac{f(0)}{F(0)} \leq \frac{2}{\eta} g\left(\frac{1}{2}\right)$,

- (Interior equilibrium) $x_{L}^{*}=\frac{1}{2}-\frac{\bar{y}}{2}$, and $x_{R}^{*}=\frac{1}{2}+\frac{\bar{y}}{2}$, if $\frac{f(1)}{F(1)}<\frac{2}{\eta} g\left(\frac{1}{2}\right)<\frac{f(0)}{F(0)}$

- (Extremism equilibrium) $x_{L}^{*}=0$ and $x_{R}^{*}=1$, if $\frac{2}{\eta} g\left(\frac{1}{2}\right) \leq \frac{f(1)}{F(1)}$.

If $F(0)=0$, the extremism equilibrium arises if and only if $\frac{2}{\eta} g\left(\frac{1}{2}\right) \leq \frac{f(1)}{F(1)}$ and the interior equilibrium arises otherwise.

Electoral advertising for each of the above SPNE is uniquely characterized in Lemma 1.

First, notice that in contrast to previous literature with similar dynamics -where equilibrium platforms require mixed strategies -Proposition (1) shows that there exists a unique pure strategy SPNE. $]^{11}$ In this unique equilibrium, the level of polarization $\left(y^{*}\right)$ can be zero (convergent equilibrium), one (extremism equilibrium) or $\bar{y}$ (interior equilibrium). The emerging type of equilibrium depends on: a) the concentration of voters around the median $g\left(\frac{1}{2}\right)$, b) the rate at which impressionable voters become ideological when polarization increases $\frac{f(y)}{F(y)}$, and c) the effectiveness of electoral campaigns $\eta$.

Starting with the effectiveness of the electoral campaigns, a large value of $\eta$ makes the competition for impressionable votes tougher, which exacerbates advertising costs in the second stage (Lemma 1). Therefore, a high value of $\eta$ provides incentives to polarize platforms in the first stage in order to reduce the number of impressionable voters. If $F(0)>0$ (i.e., there exists a share of ideological voters under convergence), as $\eta$ increases, indeed we may move across types of equilibria with convergence occurring for a larger set

\footnotetext{
${ }^{11}$ Note here that we have restricted attention to $\eta \leq 2$ due to the mixed strategies in the campaign stage for $\eta>2$. However, the unique pure strategy equilibrium characterized in the platform substage -which is the main difference to Ashworth and Bueno de Mesquita 2009- is also an equilibrium for $\eta>2$ with equilibrium platforms the same as the ones characterized for $\eta=2$. This is due to the payoff equivalence result described in the previous footnote.
} 
of parameters. But also platforms become less polarized in the interior equilibrium (i.e., $\bar{y}$ is decreasing in $\eta$ ). Similarly, when many voters are concentrated around the median (i.e., a high value of $g\left(\frac{1}{2}\right)$ ), there are strong incentives to propose moderate platforms. Thus, a strong presence of moderate voters leads to equilibria of "low" polarization (again, either across equilibria types or within the interior equilibrium). If $F(0)=0$ (i.e., no ideological voters when parties fully converge), nothing changes except that a convergent equilibrium never arises.

The conversion rate at which impressionable voters become ideological when polarization increases (i.e, the reverse hazard rate $\left.\frac{f(y)}{F(y)}\right)$ also helps understanding our result. This rate captures the incentives of increasing polarization as a way of reducing electoral advertising. By log-concavity of $F(y)$, the rate is monotonically decreasing and hence takes its maximum value at $y=0$ and its minimum value at $y=1$. If the maximum value of the conversion rate is small "enough" (i.e., $\left.\frac{f(0)}{F(0)} \leq \frac{2}{\eta} g\left(\frac{1}{2}\right)\right)$ the original Downsian result of platform convergence emerges. Despite full convergence, the conversion rate is so low that increasing polarization does not increase the share of ideological voters enough to diminish electoral advertising in a profitable fashion. Analogously, if its minimum value is large "enough" (i.e., $\left.\frac{2}{\eta} g\left(\frac{1}{2}\right) \leq \frac{f(1)}{F(1)}\right)$, polarization is very effective in restraining electoral advertising and extremism emerges. A distributional change in the function determining the distribution of voters across types gives interesting comparative statics.

Notation 1. Let $\rho$ parametrize the sensitivity of the conversion rate due to inputs other than polarization (e.g., awareness or interest in politics).

The conversion rate $f(y ; \rho) / F(y ; \rho)$ satisfies the monotone likelihood ratio property (MLRP) in $y$ if for any $\rho_{1}>\rho_{0}$ it holds that

$$
\frac{f\left(y ; \rho_{1}\right)}{F\left(y ; \rho_{1}\right)}>\frac{f\left(y ; \rho_{0}\right)}{F\left(y ; \rho_{0}\right)}
$$

where $F(y ; \rho)$ and $f(y ; \rho)$ are differentiable in $\rho$.

Any increase in $\rho$ makes the conversion rate of impressionable voters to ideological more responsive to changes in polarization. This may move platforms across types of equilibria (favoring more polarization), while also at any interior equilibrium, polarization is increasing in $\rho$ (it follows from applying implicit differentiation to the interior equilibrium condition $\left.\frac{f(\bar{y})}{F(\bar{y})}=\frac{2}{\eta} g\left(\frac{1}{2}\right)\right)$.

Finally, note that platforms' characterization does not depend on the costs of campaigning $\mu$ in any manner. Given that this cost is symmetric for the two parties, increasing or decreasing it would only rescale the equilibrium levels of advertising $e_{i}$ (from Lemma 1 ) but will not modify the actual level of campaign spending $\mu e_{i}$ and hence polarization. 


\section{Effects on Campaign Spending}

A technological change that increases the campaign effectiveness has an ambiguous effect on campaign spending. This is apparent when we look at the relevant expression:

$$
\frac{\partial \mu e_{i}^{*}\left(x_{L}^{*}, x_{R}^{*}\right)}{\partial \eta}=\overbrace{\frac{1-F\left(y^{*}\right)}{4}}^{\text {Spending effect }} \overbrace{-f\left(y^{*}\right) \frac{\partial y^{*}}{\partial \eta} \frac{\eta}{4}}^{\text {Polarization effect }}
$$

On the one hand, ceteris paribus an increase in $\eta$ increases advertising (Lemma 1). On the other hand, it also increases in equilibrium level of polarization $y^{*}$ (Proposition 1), which in turn, decreases the share of impressionable voters and so the levels of advertising (Lemma 1). We call the former the spending effect, while we label the latter as the polarization effect.

At the fully divergent and fully convergent equilibria there is no polarization effect, $\frac{\partial y^{*}}{\partial \eta}=0$, and spending either increases monotonically with $\eta$ due to the spending effect or is unaffected. At the interior equilibrium however, the polarization effect takes place and mitigates the spending effect. If polarization increases disproportionally with $\eta$, the polarization effect may even overturn the spending effect, and hence observe $\eta$ and campaign spending move in opposite directions. In Lemma (2) below, we provide the conditions for a simultaneous increase in campaign spending and polarization, and we use an example to illustrate it.

Lemma 2. In any interior equilibrium, a technological change that increases the campaign effectiveness $\eta$ - and hence polarization - also increases campaign spending due to the spending effect dominating the polarization effect if and only if effectiveness is low "enough". Formally, in any interior equilibrium $\frac{\partial \mu e_{i}^{*}\left(x_{L}^{*}, x_{R}^{*}\right)}{\partial \eta} \geq 0$ if and only if $\eta \leq 2 g\left(\frac{1}{2}\right) \frac{1-F(\bar{y})}{f(\bar{y})}\left[\frac{F(\bar{y})}{f(\bar{y})}\right]^{\prime}$.

Example 1: $F(y)$ is uniformly distributed over $(0,1]$ with a mass at zero $F(0)=\frac{1}{10}$. The "conversion rate" now - from impressionable to ideological voters is proportional to polarization: $\frac{f(y)}{F(y)}=\frac{\frac{9}{10}}{\frac{1}{10}+\frac{9}{10} y}$. From Proposition 1 , we are at a convergent equilibrium for $\eta \leq \frac{2}{9} g\left(\frac{1}{2}\right)$, at an interior equilibrium for $\frac{2}{9} g\left(\frac{1}{2}\right)<\eta<\frac{20}{9} g\left(\frac{1}{2}\right)$ and at an extremism equilibrium for $\eta \geq \frac{20}{9} g\left(\frac{1}{2}\right)$. These conditions highlight how the concentration of voters around the median gives rise to different equilibrium types. If for example $g\left(\frac{1}{2}\right)$ is large then extremism can be excluded as an outcome for any level of campaign effectiveness. In the interior equilibrium polarization given by $y^{*}=\bar{y}=\frac{\eta}{2 g\left(\frac{1}{2}\right)}-\frac{1}{9}$ and it is straightforward to see that polarization is increasing in $\eta$. Using the condition in Lemma (2) we can also get the non-monotone comparative statics on campaign spending 


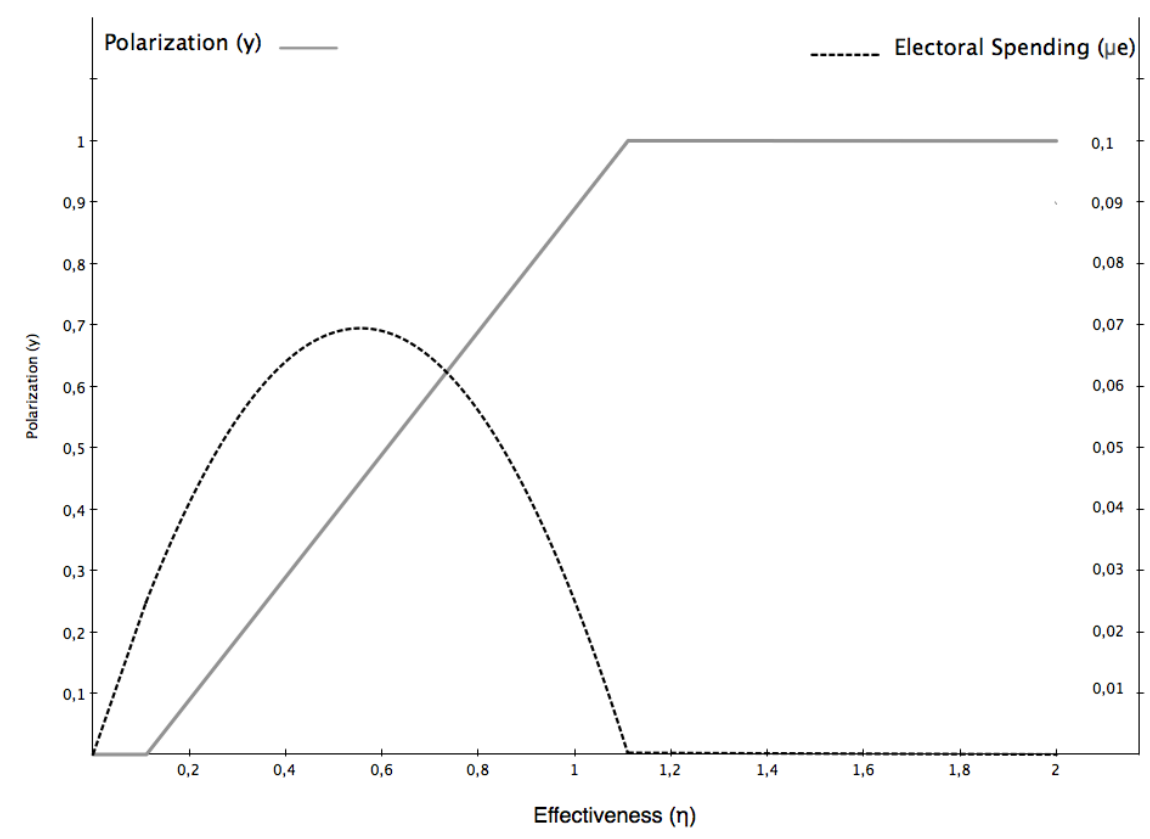

Figure 1: Comparative statics on $\eta$ for campaign spending and polarization. Uniform distribution of $F(y)$ with $F(0)=\frac{1}{10}$ and $g(1 / 2)=1 / 2$.

in the interior equilibrium and show that campaign spending is increasing in the campaign effectiveness for $\eta$ low enough (i.e., $\left.\eta \leq \frac{10}{9} g\left(\frac{1}{2}\right)\right)$ and decreasing otherwise.

In Figure 1 we graphically represent the comparative statics of changes of $\eta$ on polarization and campaign spending also assuming that $g\left(\frac{1}{2}\right)=\frac{1}{2}$. Consider first the equilibrium levels of polarization (solid line). For $\eta$ lower than $\frac{1}{9}$ or greater than $\frac{10}{9}$ the convergent and extremist equilibria respectively arise. For $\frac{1}{9}<\eta<\frac{10}{9}$ the interior equilibrium arises and polarization is strictly increasing in $\eta$. Let's now turn to campaign spending (dashed line). For $\eta \leq \frac{1}{9}$, polarization is constant and equal to zero and campaign spending is monotonically increasing in $\eta$ (as in any standard Tullock contest). If $\eta \geq \frac{10}{9}$ the extremism equilibrium arises and due to all voters being ideological campaign spending is zero ${ }^{12}$ In the interior equilibrium interval (i.e., $\eta \in\left(\frac{1}{9}, \frac{10}{9}\right)$ ), the non-monotonicity arises. Campaign spending increases until reaching $\eta=\frac{5}{9}$ due to the spending effect being larger than the polarization one. On the contrary, spending decreases for values of $\eta$ larger than $\frac{5}{9}$ due to the polarization effect overcoming the spending effect and till extremism arises.

Polarization and campaign spending are also affected by the rate at which impressionable voters become ideological as polarization increases parametrized by $\rho$. A technolog-

12 Zero spending under extremism arises because of the simplification of no mass of impressionable voters under maximal platform separation. One could trivially extend our model by including a mass of impressionable voters $0<\delta<1$ even under extreme polarization. In that case, in the extreme equilibrium campaign spending is positive and strictly increasing in $\eta$. Our characterization in Proposition 1 would remain unaffected and advertising levels in Lemma 1 will be simply rescaled but not qualitatively affected. 
ical change that increases $\rho$ has an ambiguous effect on campaign spending. As above, the condition comes from looking at the derivative of campaign spending with respect to $\rho$.

$$
\frac{\partial \mu e_{i}^{*}\left(x_{L}^{*}, x_{R}^{*}\right)}{\partial \rho}=\overbrace{-\frac{\partial F\left(y^{*}\right)}{\partial \rho} \frac{\eta}{4}}^{\text {Spending effect (+) Polarization effect (-) }} \overbrace{-\frac{\partial F\left(y^{*}\right)}{\partial y^{*}} \frac{\partial y^{*}}{\partial \rho} \frac{\eta}{4}}^{\text {Pon }}
$$

On the one hand, an increase in $\rho$ increases the "stock" of impressionable voters $1-F(y)$ (this follows directly from MLRP which implies first order stochastic dominance). The spending effect then suggests that, for a given level of polarization $y$, an increase in $\rho$ increases advertising (Lemma 1). On the other hand, an increase in $\rho$ makes voters more responsive to polarization, by affecting the "conversion" rate $\frac{f(y)}{F(y)}$, and therefore provides incentives to increase polarization (Proposition 1). But this increased polarization in turn, decreases the share of impressionable voters and so decreases the levels of advertising (Lemma 1). As before, we label this latter effect as the polarization effect.

At the convergent equilibria there is no polarization effect since $\frac{\partial y^{*}}{\partial \rho}=0$ and spending increases monotonically with $\rho$. That is, an increase in $\rho$ keeping polarization constant would increase the number of impressionable voters and their weight in the parties' maximization problem and parties would have higher incentives to increase advertising. In the interior equilibrium the polarization effect kicks in and the net effect on spending depends on the magnitude of these two effects. Recall that changes in $\rho$ enter in the spending effect due to changes in "stock", while they enter in the polarization effect due to changes in the "conversion". Finally, polarization is constant in $\rho$ once the extremism equilibrium is reached and spending is constant and equal to zero since there are no impressionable voters. The following lemma summarizes the above for the interior equilibrium.

Lemma 3. In any interior equilibrium, a technological change that increases $\rho$ - and hence polarization - also increases campaign spending due to the spending effect dominating the polarization effect if and only if the effect of $\rho$ on the "stock" of impressionable voters is "large" enough. Formally, in any interior equilibrium $\frac{\partial \mu e_{i}^{*}\left(x_{L}^{*}, x_{R}^{*}\right)}{\partial \rho} \geq 0$ if and only if $-\frac{\partial F(\bar{y})}{\partial \rho} \geq f(\bar{y}) \frac{\partial \bar{y}}{\partial \rho}$.

Finally, notice that in the symmetric case, the marginal cost of advertising plays no role in equilibrium: neither the platforms nor the campaign spending depend on $\mu$. When we introduce a cost-asymmetry between the parties, the equilibrium changes, favoring the party with the lower marginal cost. 


\subsection{Asymmetric parties}

In contrast to previous models with endogenous valence, we can incorporate cost asymmetries and obtain results of similar intuition. In this section we assume, without loss of generality, that $\mu_{L}<\mu_{R}$. We first characterize campaign spending in the second stage and then show how equilibrium platforms are affected by the asymmetry. Following Baik (1994) and Nti (1999) the following Lemma arises.

Lemma 4. Let $\bar{\eta}$ implicitly defined by $\mu_{L}^{\bar{\eta}}+\mu_{R}^{\bar{\eta}}=\bar{\eta} \mu_{R}^{\bar{\eta}}$. For all $\eta \leq \bar{\eta}$ there exists a unique Nash equilibrium in the campaign stage and advertising is given by $e_{i}^{*}\left(x_{L}, x_{R}\right)=$ $(1-F(y)) \frac{\eta}{\mu_{i}} \frac{\mu_{L}^{\eta} \mu_{R}^{\eta}}{\left(\mu_{L}^{\eta}+\mu_{R}^{\eta}\right)^{2}}$ for all $i$.

Lemma (4) shows that, in equilibrium, parties choose different levels of advertising $\left(e_{i}^{*}\left(x_{L}, x_{R}\right)\right)$ although spend equal amounts $\left(\mu_{i} e_{i}^{*}\left(x_{L}, x_{R}\right)\right){ }^{13}$ The share of impressionable voters is not any longer equally split across parties, giving an advantage to the party with the lower marginal cost. This generates an asymmetry in parties' incentives to use polarization as a device to reduce campaign spending and eliminates the interior equilibria where $x_{L}^{*}+x_{R}^{*}=1$. In any interior equilibrium parties propose asymmetric platforms. The convergent and extremism equilibria described previously also arise. We present the conditions for the rise of each type of equilibrium in the following propositions.

Proposition 2. (Convergent equilibrium) For any $\eta \leq \bar{\eta}, \mu_{L}<\mu_{R}$ and $F(0)>0$, there exists a unique SPNE with $x_{L}^{*}=x_{R}^{*}=\frac{1}{2}$ if and only if $\frac{f(0)}{F(0)} \leq g\left(\frac{1}{2}\right) /\left(\frac{\mu_{L}^{\eta} \mu_{R}^{\eta}}{\left(\mu_{L}^{\eta}+\mu_{R}^{\eta}\right)^{2}} 2 \eta+\frac{\mu_{R}^{\eta}-\mu_{L}^{\eta}}{\mu_{L}^{\eta}+\mu_{R}^{\eta}}\right)$. Campaign spending for the convergent equilibrium is uniquely characterized in Lemma 4.

As in the symmetric case, the convergent equilibrium arises when a lot of voters are concentrated around the median (i.e., high $\left.g\left(\frac{1}{2}\right)\right)$ and/or the conversion rate at $y=0$ (i.e., at its maximal level $\frac{f(0)}{F(0)}$ ) is low. In the asymmetric case, the size of the asymmetry is also a determinant of platform convergence. If the asymmetry converges to zero, then the inequality characterizing a convergent equilibrium converges to the one of Proposition 1. As the asymmetry however increases, platform convergence becomes less likely (the denominator at the right hand side of the inequality is increasing in the asymmetry). This is because, as the asymmetry increases, the (symmetric) convergent equilibrium becomes less attractive for the disadvantaged party that loses the competition for impressionable voters and hence has incentives to diversify and propose distinct platforms. This potentially leads to an interior equilibrium.

\footnotetext{
${ }^{13}$ The condition on $\eta$ that guarantees equilibrium in pure strategies is more restrictive than in the symmetric case, since $\bar{\eta}$ is lower or equal than 2. For $\eta \in(\bar{\eta}, 2)$ the equilibrium is characterized by Wang (2010) and Ewerhart (2017), while payoff equivalence by Alcalde and Dahm (2010) can be used to solve the platform stage for $\eta>2$.
} 
An equilibrium is interior if at least one of the parties chooses an interior platform. That is, a platform between the median and the corner (either 0 or 1, depending on the party). Taking this definition into account, Proposition 3 describes the necessary and sufficient conditions for the existence and uniqueness of an interior equilibrium, which depend on the extent of the parties' cost asymmetries. Then, within the scope of the proposition, the corollary below characterizes the interior equilibria.

Proposition 3. (Interior Equilibrium) For any $\eta \leq \bar{\eta}$ and $\mu_{L}<\mu_{R}$, let $\bar{x}^{*}=G^{-1}\left(\frac{\mu_{R}^{\eta}}{\mu_{L}^{\eta}+\mu_{R}^{\eta}}\right)$ and $\bar{y}$ be implicitly defined by $\frac{f(\bar{y})}{F(\bar{y})}=\frac{g\left(\bar{x}^{*}\right)}{2 \eta} \frac{\left(\mu_{L}^{\eta}+\mu_{R}^{\eta}\right)^{2}}{\mu_{L}^{\eta} \mu_{R}^{\eta}}$, there exists a unique interior SPNE if and only if one of the following conditions is satisfied

$$
\begin{aligned}
& \frac{F\left(2 \bar{x}^{*}-1\right)}{f\left(2 \bar{x}^{*}-1\right)} g\left(\bar{x}^{*}\right)<2 \eta \frac{\mu_{L}^{\eta} \mu_{R}^{\eta}}{\left(\mu_{L}^{\eta}+\mu_{R}^{\eta}\right)^{2}}<\frac{F(1)}{f(1)} g(0.5)+\frac{\mu_{R}^{\eta}-\mu_{L}^{\eta}}{\mu_{R}^{\eta}+\mu_{L}^{\eta}} \quad \text { if } \quad \frac{\mu_{R}^{\eta}}{\mu_{L}^{\eta}+\mu_{R}^{\eta}}<G\left(\frac{3}{4}\right) \\
& \frac{F\left(\frac{1}{2}\right)}{f\left(\frac{1}{2}\right)} g\left(\frac{1}{2}\right)-2 G\left(\frac{3}{4}\right)+\frac{2 \mu_{R}^{\eta}}{\mu_{R}^{\eta}+\mu_{L}^{\eta}}<2 \eta \frac{\mu_{L}^{\eta} \mu_{R}^{\eta}}{\left(\mu_{L}^{\eta}+\mu_{R}^{\eta}\right)^{2}} \quad \text { if } \frac{\mu_{R}^{\eta}}{\mu_{L}^{\eta}+\mu_{R}^{\eta}} \geq G\left(\frac{3}{4}\right)
\end{aligned}
$$

Corollary 1. (Characterization of Interior Equilibria) There are two types of Interior equilibria. Proposition 3 describes the necessary and sufficient conditions for existence and uniqueness. Their characterization is as follows:

- (Interior/Interior) $x_{L}^{*}=\bar{x}^{*}-\frac{\bar{y}}{2}$ and $x_{R}^{*}=\bar{x}^{*}+\frac{\bar{y}}{2}$ if and only if Condition 3 and $2 \eta \frac{\mu_{L}^{\eta} \mu_{R}^{\eta}}{\left(\mu_{L}^{\eta}+\mu_{R}^{\eta}\right)^{2}}<\frac{F\left(2-2 \bar{x}^{*}\right)}{f\left(2-2 \bar{x}^{*}\right)} g\left(\bar{x}^{*}\right)$ are satisfied.

- (Interior/Corner) $x_{L}^{*}<0.5$ and $x_{R}^{*}=1$, otherwise.

Campaign spending for each of the above SPNE is uniquely characterized in Lemma 4

All interior equilibria are asymmetric. It can be that both parties propose interior platforms (Interior/Interior) or that the cost advantaged party proposes an interior platform while the disadvantaged party proposes an extreme platform (Interior/Corner). The cost asymmetry plays an important role in determining the type but also polarization level at the interior equilibrium. The cost disadvantaged party $R$ has more incentives than the advantaged party to reduce the share of (costly) impressionable voters. Consequently, $R$ has more incentives to separate its platform from $L$. Thus, in any interior equilibrium platforms are shifted towards the cost-disadvantaged party $R$. That is, in the (Interior/Interior) equilibrium the point around which parties propose equidistant platforms is to the right (i.e., $\bar{x}^{*}>0.5$ ), while in the (Interior/Corner) it is the disadvantaged party that proposes an extreme platform. Note that in general, if the asymmetry is low "enough" (i.e., (3) is satisfied) the disadvantaged party may also propose an interior platform. If this asymmetry however is high "enough" (i.e., (4) is satisfied) only the (Interior/Corner) equilibrium arises, the disadvantaged party would like to separate more but cannot due to having reached the corner. The disadvantaged party best responds 
to that by proposing an interior platform. In that sense, "large" asymmetries never give rise to extremism equilibria either as the following proposition summarizes.

Proposition 4. (Extremism Equilibrium) For any $\eta \leq \bar{\eta}$ and $\mu_{L}<\mu_{R}$, there exists a unique SPNE with $x_{L}^{*}=0$ and $x_{R}^{*}=1$ if and only if $g(0.5) /\left(2 \eta \frac{\mu_{L}^{\eta} \mu_{R}^{\eta}}{\left(\mu_{L}^{\eta}+\mu_{R}^{\eta}\right)^{2}}-\frac{\mu_{R}^{\eta}-\mu_{L}^{\eta}}{\mu_{L}^{\eta}+\mu_{R}^{\eta}}\right) \leq \frac{f(1)}{F(1)}$ and campaign spending as characterized in Lemma 4.

If the asymmetry converges to zero, the relevant inequality converges to the one of Proposition 1. As the asymmetry however increases, extremism becomes less likely (the denominator on the left hand side of the inequality is decreasing in the asymmetry). This is because, the advantaged party has no incentives to polarize as it would reduce the share of impressionable voters for whom it enjoys a great advantage. Also, as in the symmetric case, extremism is more likely when the rate at which impressionable voters become ideological is great enough under maximal platform separation (i.e., $\frac{f(1)}{F(1)}$ is high).

As with the symmetric case, the inequalities in all equilibrium conditions also depend on the effectiveness of campaigns, the mass of ideological voters at the median and conversion rates at different points. While we have covered the most relevant ones, especially what concerns the cost asymmetries, in general, the incentives to polarize (or not) and move across equilibria types remain similar to the symmetric case. Proceeding with examples, similar intuition to the results of the symmetric case arise. The simultaneous increase in polarization and campaign spending observed can be reconciled also in the presence of an asymmetry.

Example 2: $F(y)$ and $\mathbf{G}(\mathbf{y})$ uniformly distributed in $X$. In Figure 2 Panel (a) we plot the case of "low" asymmetry $\left(\mu_{R} / \mu_{L}=1.2\right)$ while in Panel (b) we plot the case of "high" asymmetry $\left(\mu_{R} / \mu_{L}=2\right)$. For both levels of asymmetry, we are at a convergent equilibrium only when $\eta=0$. That is, when impressionable voters are split equally across the two parties regardless of electoral advertising parties do not have incentives to differentiate their platforms since the asymmetry vanishes. As long as $\eta>0$ we illustrate the divergent and asymmetric (Interior/Interior) equilibrium platforms as characterized in Corollary 1. Note that in both panels, and as described in our results, the disadvantaged party $R$ is proposing a relatively more extreme platform compared to the advantaged party $L$. In the example of "low" asymmetry, platforms diverge monotonically as $\eta$ increases (as in the symmetric case) and hence polarization is also increasing. In the example of "high" asymmetry, and despite polarization being again increasing in the effectiveness of electoral advertising, the advantaged party does not respond to increases in $\eta$ in a monotonic manner. Finally, when it comes to campaign spending, as the upper right panel shows, we may again encounter a situation of a non-monotonic relationship 
between campaign spending and $\eta$ (as in Figure 1 and the symmetric case). But perhaps more importantly, our results can again sustain the simultaneous increase in polarization and campaign spending due to technological changes even in the presence of asymmetries for a wide range of parameters.

Panel (a): "low" asymmetry: $\mu_{R} / \mu_{L}=1.2$
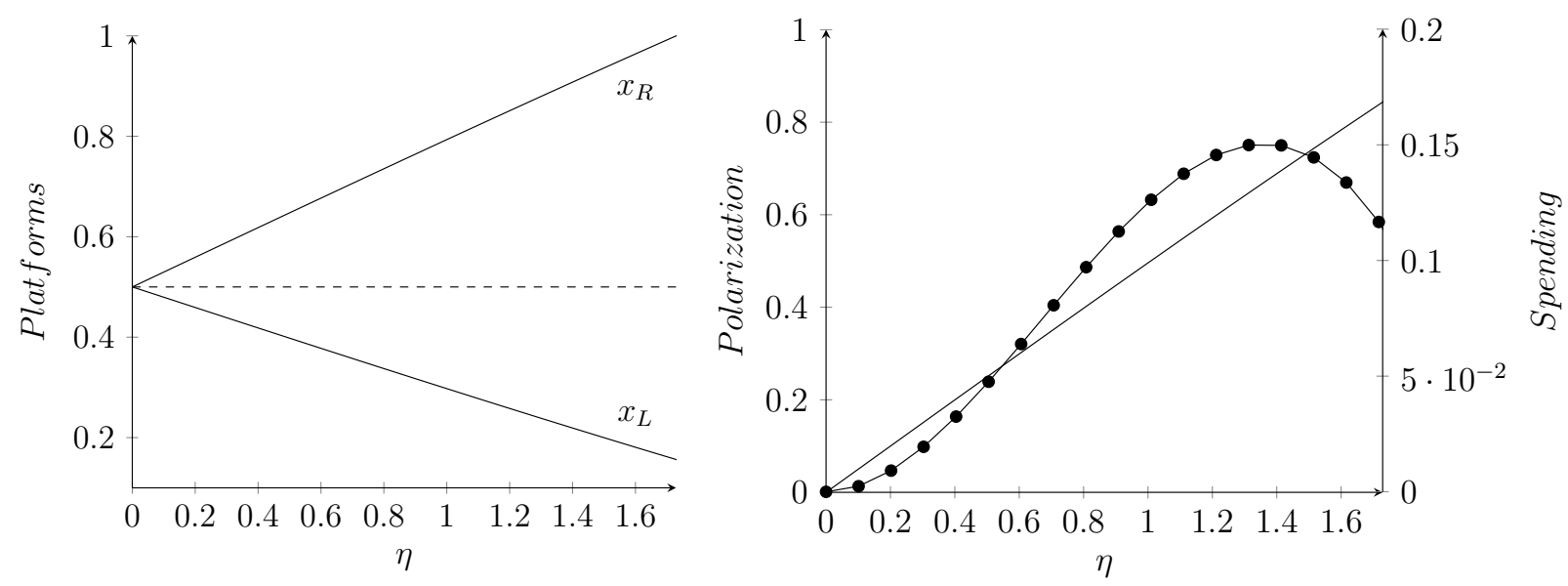

Panel (b): "high" asymmetry: $\mu_{R} / \mu_{L}=2$
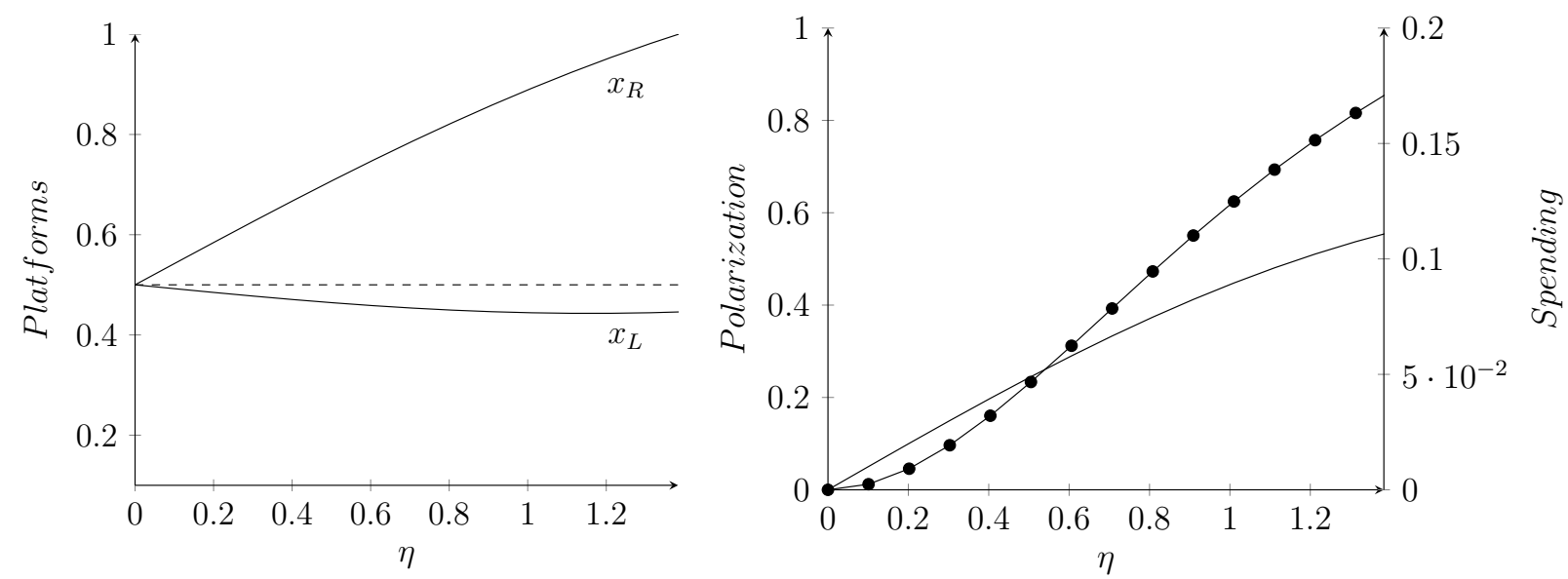

Figure 2: Solid lines depict equilibrium platforms $\left(x_{L}^{*}, x_{R}^{*}\right)$ in the left panels and polarization $\left(x_{R}^{*}-x_{L}^{*}\right)$ in the right panels. Dotted lines depict campaign spending. Graphs are plotted on the interval of $\eta$ that guarantees an equilibrium in pure strategies in the advertising stage.

\section{Conclusion}

Citing Herrera et al. (2008), "commentators have suggested that the reason for both the increased polarization and campaign spending is that skilled political operatives using sophisticated statistical tools and purchasing advertising in local markets are better able to 
target particular voters" (see for example, NBC 2017). However, existing results so far were linking such technological advances with a reduction in polarization, and therefore favored alternative channels that may drive polarization such as more volatile preferences. Our results in contrast, are the first to justify the simultaneous increase in polarization and campaign spending due to recent technological changes and better targeting of the electoral campaigns.

Given that one would naturally expect further advances in campaign technology two natural questions arise: a) should we expect a further increase in polarization?, and b) what about campaign spending? Our theory would say yes, further advances in targeting will lead to further polarization. Ways to go against this trend would require policies that improve the awareness of the electorate and a shift of voters' attention from persuasive campaigns to political platforms. With regards to campaign spending, recall that our theory does not provide a monotone comparative static. As the targeting technology improves, parties have incentives to increase their campaign spending, but at the same time to polarize (which reduces campaign spending). Hence, our results are not incompatible with the observations of the 2016 presidential US election where campaign spending dropped. Furthermore, following this presidential campaign, the diffusion of plausible but false information received the name of "fake news". This notion, widely used during and after the campaigns, has direct implications on the electorate's awareness: for any given "real" platforms, the stock of ideological voters decreases with the amount of fake news. In terms of our model, fake news would increase the reversed hazard rate, causing an increase in polarization as well.

While one of our contributing messages could be the importance of the electorate's awareness as a way to affect campaign spending and polarization, political pundits have paid special attention to caps on campaign spending. In the context of symmetric costs, our model provides very clear and intuitive implications regarding the effects of this policy. If a cap is below the equilibrium campaign spending, it will completely shut down the polarization effect in our model. That is, when a cap is binding, the parties will not induce an increase in campaign spending by moving their platform towards their competitor. This eliminates one of the elements of the parties' trade-off in the choice of platform location. As a consequence, platform convergence is the only possible outcome. In other words, any cap on campaign spending smaller or equal to the equilibrium spending, will also induce convergence at the median voter. Thus our model predicts an important impact of campaign caps on party polarization. The same intuition can be sustained under asymmetric costs if the inverse hazard rate is great enough under full convergence. 


\section{Appendix - Proofs}

This section is organized as follows. In Subsection 5.1 we show the pure strategy equilibrium in advertising for asymmetric costs. Additionally, we discuss the extension for $\eta>\bar{\eta}$ in the symmetric case. In Subsection 5.2 we write and solve the platform selection problem for the general case with asymmetric costs. Thus, taking into account the Kuhn-Tucker conditions, we solve this maximization problem and we show the first and second order conditions. Lemmi A.1, A.2 and Remark 1 in that subsection provide the conditions for existence and uniqueness of platforms that maximize the Lagrangian for the general case. Next, we show uniqueness and existence of a unique (pure) Subgame Perfect Nash Equilibrium, and we characterize the equilibrium platforms for the symmetric case (Proposition 1). Later on, we do the same for asymmetric costs. In this case, we show separately the convergent equilibrium (Proposition 2), the interior equilibrium (Proposition 3 and Corollary 1) and the extremism one (Proposition 4).

\subsection{Advertising}

We begin proving the more general Lemma 4 , i.e., $\mu_{L} \neq \mu_{R}$, and after the proof we discuss the implications of symmetry, i.e., Lemma 1.

Proof. For any given pair $\left(x_{L}, x_{R}\right)$, parties simultaneously choose $e_{i}$ that maximizes $(1-$ $F(y)) \frac{e_{i}^{\eta}}{e_{L}^{\eta}+e_{R}^{\eta}}-\mu_{i} e_{i}\left(x_{L}, x_{R}\right)$. Hence, from the FOC, we obtain the first necessary condition for an interior PSNE: $e_{i}=\frac{1-F(y)}{\mu_{i}} \eta \frac{\mu_{L}^{\eta} \mu_{R}^{\eta}}{\left(\mu_{L}^{\eta}+\mu_{R}^{\eta}\right)^{2}}$. The equilibrium payoffs can be written as

$$
(1-F(y))\left[\frac{\mu_{i}^{\eta}}{\mu_{L}^{\eta}+\mu_{R}^{\eta}}-\eta \frac{\mu_{L}^{\eta} \mu_{R}^{\eta}}{\left(\mu_{L}^{\eta}+\mu_{R}^{\eta}\right)^{2}}\right]
$$

which are positive if and only if $\left(\mu_{L}^{\eta}+\mu_{R}^{\eta}\right) \mu_{i}^{\eta}-\eta \mu_{L}^{\eta} \mu_{R}^{\eta} \geq 0$. Therefore, the second necessary condition for PSNE is that $\eta \leq \bar{\eta}: \mu_{R}^{\bar{\eta}}+\mu_{L}^{\bar{\eta}}=\bar{\eta} \mu_{R}^{\bar{\eta}}$. Moreover, for all $\eta$ lower or equal than $\bar{\eta}$, the SOC holds with strict inequality, assuring not only existence but also uniqueness of the equilibrium.

The symmetric case. Notice that $\bar{\eta} \leq 2$ holds with strict equality if and only if $\mu_{L}=\mu_{R}$. In the symmetric case, for all $\eta \leq 2$, the conditions on the existence and uniqueness of PSNE are satisfied, and therefore $e_{i}=\frac{1-F(y)}{4 \mu_{i}} \eta$. For $\eta>2$, Alcalde and Dahm (2010) show that "the contest possesses an all-pay auction equilibrium" (Theorem 3.2 in their paper). In a symmetric contest, this theorem implies full dissipation, which means that the expected payoff would be 0 for parties $L$ and $R$, and the corresponding expected bids would be $\frac{1}{2} \frac{1-F(y)}{\mu_{i}} \eta$. 


\subsection{Polarization and Spending}

\section{The objective function, existence and uniqueness}

Throughout this section let $y=x_{R}-x_{L}, \bar{x}=\frac{x_{R}+x_{L}}{2}$, and $S_{I d l}^{L}\left(x_{L}, x_{R}\right)=G(\bar{x})=1-$ $S_{I d l}^{R}\left(x_{L}, x_{R}\right)$ for $x_{L} \neq x_{R}$ and $S_{I d l}^{L}\left(x_{L}, x_{R}\right)=S_{I d l}^{R}\left(x_{L}, x_{R}\right)=\frac{1}{2}$ otherwise. By backward induction and using the equilibrium expressions of the of advertising subgame, we can write the first-stage payoff for the political parties as follows

$$
\Pi_{i}\left(x_{L}, x_{R}\right)=F(y) S_{I d l}^{i}\left(x_{L}, x_{R}\right)+(1-F(y)) \frac{\mu_{-i}^{\eta}}{\mu_{R}^{\eta}+\mu_{L}^{\eta}}-(1-F(y)) \eta \frac{\mu_{R}^{\eta} \mu_{L}^{\eta}}{\left(\mu_{R}^{\eta}+\mu_{L}^{\eta}\right)^{2}} .
$$

Lemma A.1. In any equilibrium $x_{L} \leq 1 / 2$ and $x_{R} \geq 1 / 2$.

Proof. Remind that we have assumed without loss of generality that $x_{L} \leq x_{R}$. First consider a divergent equilibrium, i.e., $x_{L} \neq x_{R}$. We proceed by contradiction. Assume an equilibrium such that $1 / 2<\tilde{x}_{L}<\tilde{x}_{R}$. Then party $R$ is strictly better of by deviating to $x_{R}=2 \tilde{x}_{L}-\tilde{x}_{R}$, which maintains unchanged the proportion of each type of voter and the share of impressionable votes and strictly increases the share of ideological votes of party $R$. Analogously we can show that party $L$ always has a profitable deviation to $x_{L}=2 \tilde{x}_{R}-\tilde{x}_{L}$ when $\tilde{x}_{L}<\tilde{x}_{R}<1 / 2$

Now consider a convergent equilibrium $x_{L}=x_{R}$. Given the assumption of $x_{L} \leq x_{R}$ two necessary conditions for a convergent equilibrium at $x_{L}=x_{R}=x$ are:

$$
\begin{aligned}
& \lim _{x_{L} \rightarrow x^{-}} \Pi_{L}\left(x_{L}, x\right) \leq \Pi_{L}(x, x) \\
& \lim _{x_{R} \rightarrow x^{+}} \Pi_{R}\left(x, x_{R}\right) \leq \Pi_{R}(x, x)
\end{aligned}
$$

For $x>\frac{1}{2}, \lim _{x_{L} \rightarrow x^{-}} G\left(x_{L}, x\right)>\frac{1}{2}=G(x, x)$, which implies that $\lim _{x_{L} \rightarrow x^{-}} \prod_{L}\left(x_{L}, x\right)>$ $\Pi_{L}(x, x)$ and violates the equilibrium condition for party $L$. A similar argument applies for party $R$ if $x<\frac{1}{2}$.

Remind that when $x_{L}=x_{R} \neq 1 / 2$, a discontinuity can arise in the objective function, due to the discontinuity of $S_{I d l}^{i}(x)$. However, the previous lemma restricts convergence to the case $x_{L}=x_{R}=0.5$, in which $S_{I d l}^{i}(x)$, and consequently $\Pi_{i}\left(x_{L}, x_{R}\right)$, are continuous. Thus, by including the equilibrium constraints $\left(x_{L} \leq 1 / 2\right.$ and $\left.x_{R} \geq 1 / 2\right)$ in the maximization problem, the Lagrangians below are continuous, and provided that the second order conditions are met, will be solved by the equilibrium platforms (due to the presence of equilibrium constraints solving the Lagrangian is a necessary but not a sufficient condition for equilibrium, as we show in Remark 1 below).

For $i \in\{L, R\}$, let $\lambda_{i} \geq 0$ be the multipliers associated with the feasibility constraints 
and $\nu_{i} \geq 0$ with the equilibrium constraints. The Lagrangians are:

$$
\begin{aligned}
& \mathcal{L}_{L}=\Pi_{L}\left(x_{L}, x_{R}\right)-\lambda_{L}\left(-x_{L}-0\right)-\nu_{L}\left(x_{L}-\frac{1}{2}\right) \\
& \mathcal{L}_{R}=\Pi_{R}\left(x_{L}, x_{R}\right)-\lambda_{R}\left(x_{R}-1\right)-\nu_{R}\left(\frac{1}{2}-x_{R}\right) .
\end{aligned}
$$

The first order conditions (FOC from now on) are:

$$
\begin{aligned}
\frac{\partial \mathcal{L}_{L}}{\partial x_{L}} & =\frac{\partial \prod_{L}\left(x_{L}, x_{R}\right)}{\partial x_{L}}+\lambda_{L}-\nu_{L}=0 \\
\frac{\partial \mathcal{L}_{R}}{\partial x_{R}} & =\frac{\partial \prod_{R}\left(x_{L}, x_{R}\right)}{\partial x_{R}}-\lambda_{R}+\nu_{R}=0 .
\end{aligned}
$$

Where,

$$
\begin{aligned}
& \Pi_{L}^{\prime} \equiv \frac{\partial \Pi_{L}\left(x_{L}, x_{R}\right)}{\partial x_{L}}=F(y) \frac{g(\bar{x})}{2}-f(y) G(\bar{x})+f(y) \frac{\mu_{R}^{\eta}}{\mu_{R}^{\eta}+\mu_{L}^{\eta}}-f(y) \eta \frac{\mu_{R}^{\eta} \mu_{L}^{\eta}}{\left(\mu_{R}^{\eta}+\mu_{L}^{\eta}\right)^{2}}, \\
& \Pi_{R}^{\prime} \equiv \frac{\partial \Pi_{R}\left(x_{L}, x_{R}\right)}{\partial x_{R}}=-F(y) \frac{g(\bar{x})}{2}+f(y)[1-G(\bar{x})]-f(y) \frac{\mu_{L}^{\eta}}{\mu_{R}^{\eta}+\mu_{L}^{\eta}}+f(y) \eta \frac{\mu_{R}^{\eta} \mu_{L}^{\eta}}{\left(\mu_{R}^{\eta}+\mu_{L}^{\eta}\right)^{2}}
\end{aligned}
$$

Lemma A.2. Let $F(x)$ be log-concave and $g(x)$ be symmetric and log-concave. The objective functions are strictly quasiconcave in the policy space when $x_{L} \leq \frac{1}{2} \leq x_{R}$, hence the second order conditions (SOC) are satisfied.

The constrained optimization problem includes two linear constraints for each party, thus focusing on the quasiconcavity of the payoff functions suffices.

Proof. Without loss of generality, consider party $L$. Under the conditions of Lemma (A.1), continuity of $\Pi_{L}$ is assured. Then, let us modify Equation (9) by dividing it over the densities $f(y)$ and $g(\bar{x})$ :

$$
\tilde{\Pi}_{L}^{\prime} \equiv \frac{\Pi_{L}^{\prime}}{f(y) g(\bar{x})}=\frac{F(\bar{y})}{2 f(\bar{y})}-\frac{1}{g(\bar{x})}\left[G(\bar{x})-\frac{\mu_{R}^{\eta}}{\mu_{R}^{\eta}+\mu_{L}^{\eta}}+\eta \frac{\mu_{R}^{\eta} \mu_{L}^{\eta}}{\left(\mu_{R}^{\eta}+\mu_{L}^{\eta}\right)^{2}}\right]
$$

Let $\tilde{\Pi}_{L}$ be the primitive of $\tilde{\Pi}_{L}^{\prime}$. $\tilde{\Pi}_{L}$ is strictly quasiconcave if and only if $\tilde{\Pi}_{L}^{\prime}(x)\left(x^{\prime}-x\right)>0$ whenever $\tilde{\Pi}_{L}\left(x^{\prime}\right)>\tilde{\Pi}_{L}(x)$. Since strict quasiconcavity is determined by the sign of $\tilde{\Pi}_{L}^{\prime}(x)$, which is the same of the sign of $\Pi_{L}^{\prime}(x)$ (because $f(y) g(\bar{x})$ is strictly positive), the strict quasiconcavity of $\tilde{\Pi}_{L}(x)$ guarantees the strict quasiconcavity of $\Pi_{L}(x)$.

Therefore, by showing the strict concavity of $\tilde{\Pi}_{L}$ (i.e., $\left.\tilde{\Pi}_{L}^{\prime \prime}=\frac{\partial \frac{\Pi_{L}^{\prime}}{f(y) g(\bar{x})}}{\partial x_{L}}<0\right)$, we will be proving that $\Pi_{L}\left(x_{L}, x_{R}\right)$ is strictly quasiconcave too. Hence $\tilde{\Pi}_{L}^{\prime \prime}$ is

$$
\frac{\partial \frac{\Pi_{L}^{\prime}}{f(y) g(\bar{x})}}{\partial x_{L}}=-\frac{1}{2}\left[\frac{F(\bar{y})}{f(\bar{y})}\right]^{\prime}-\frac{1}{2 g(\bar{x})^{2}}\left\{g(\bar{x})^{2}-g^{\prime}(\bar{x})\left[G(\bar{x})-\frac{\mu_{R}^{\eta}}{\mu_{R}^{\eta}+\mu_{L}^{\eta}}+\eta \frac{\mu_{R}^{\eta} \mu_{L}^{\eta}}{\left(\mu_{R}^{\eta}+\mu_{L}^{\eta}\right)^{2}}\right]\right\}
$$


By log-concavity of $F(y)$, the term $-\left[\frac{F(\bar{y})}{2 f(\bar{y})}\right]^{\prime}$ is negative, so we can focus on the negativity of $H=-\left\{g(\bar{x})^{2}-g^{\prime}(\bar{x})\left[G(\bar{x})-\frac{\mu_{R}^{\eta}}{\mu_{R}^{\eta}+\mu_{L}^{\eta}}+\eta \frac{\mu_{R}^{\eta} \mu_{L}^{\eta}}{\left(\mu_{R}^{\eta}+\mu_{L}^{\eta}\right)^{2}}\right]\right\}$ in the expression above to guarantee strict concavity of $\tilde{\Pi}_{L}(x)$ (and hence strict quasi-concavity of $\Pi_{L}(x)$ ). Let us consider two cases.

- If $g^{\prime}(\bar{x}) \geq 0$, then $\left.-g^{\prime}(\bar{x})\left[\frac{\mu_{R}^{\eta}}{\mu_{R}^{\eta}+\mu_{L}^{\eta}}-\eta \frac{\mu_{R}^{\eta} \mu_{L}^{\eta}}{\left(\mu_{R}^{\eta}+\mu_{L}^{\eta}\right)^{2}}\right]\right)$ is negative (strictly negative for $g^{\prime}(\bar{x})>$ 0 ) because the term in brackets is always positive given $\eta<\bar{\eta}$ (see proofs of Lemmi 1 and 4 . Log-concavity of $g(x)$ implies log-concavity of $G(x)$, thus - $\left[g(\bar{x})^{2}-\right.$ $\left.g^{\prime}(\bar{x}) G(\bar{x})\right]$ is negative (strictly negative for $g^{\prime}(\bar{x})=0$ ). Hence $H$ is strictly negative and $\Pi_{L}(x)$ strictly quasi-concave.

- If $g^{\prime}(\bar{x})<0$, suppose there exists $\hat{x}_{L}: G\left(\frac{\hat{x}_{L}+x_{R}}{2}\right)=\frac{\mu_{R}^{\eta}}{\mu_{R}^{\eta}+\mu_{L}^{\eta}}-\eta \frac{\mu_{R}^{\eta} \mu_{L}^{\eta}}{\left(\mu_{R}^{\eta}+\mu_{L}^{\eta}\right)^{2}}$. Since $G(x)$ is increasing in $x$, for $x_{L}>\hat{x}_{L}, H$ would be strictly negative and $\Pi_{L}(x)$ strictly quasi-concave. For $x_{L} \leq \hat{x}_{L}, G(\bar{x}) \leq \frac{\mu_{R}^{\eta}}{\mu_{R}^{\eta}+\mu_{L}^{\eta}}-\eta \frac{\mu_{R}^{\eta} \mu_{L}^{\eta}}{\left(\mu_{R}^{\eta}+\mu_{L}^{\eta}\right)^{2}}$ implies that $\frac{\partial \Pi_{L}\left(x_{L}, x_{R}\right)}{\partial x_{L}}$ is strictly positive which directly implies that $\Pi_{L}\left(x_{L}, x_{R}\right)$ is strictly quasiconcave for $x_{L} \in\left[0, \hat{x}_{L}\right]$.

We can proceed similarly to show that $\Pi_{R}\left(x_{L}, x_{R}\right)$ is also strictly quasiconcave (in that case we use that $g(x)$ implies that $1-G(x)$ is log-concave).

Remark 1. Let $\left(x_{L}, x_{R}\right)$ be a solution to the Lagrangians. Then, in the platforms stage, there is a divergent equilibrium $\left(x_{L} \neq x_{R}\right)$ only when $\nu_{L}=\nu_{R}=0$ and there is a convergent one $\left(x_{L}=x_{R}\right)$ only when $\nu_{L} \geq 0$ and $\nu_{R} \geq 0$.

Proof. For the divergent equilibria, let $x_{L} \neq x_{R}$ be an equilibrium with $\nu_{R}=0$. Suppose $\nu_{L}>0$. Then $x_{L}=\frac{1}{2}<x_{R}$ and $\Pi_{L}^{\prime}\left(\frac{1}{2}, x_{R}\right)>0$. Thus, party $L$ has incentives to deviate to a platform strictly larger than $\frac{1}{2}$ which violates Lemma A.1). Then $x_{L} \neq x_{R}$ with $\nu_{R}=0<\nu_{L}$ cannot be an equilibrium. Hence, $\nu_{L}$ must be 0 for this type of equilibrium to exist. The same holds true for $\nu_{L}=0<\nu_{R}$.

For the convergent equilibrium, Lemma A.1 implies $x_{L}=x_{R}=\frac{1}{2}$ is the only candidate to equilibrium. Hence, at the solution to the Lagrangian, $\nu_{L} \geq 0$ and $\nu_{R} \geq 0$ is true. Notice that even if $\Pi_{L}^{\prime}\left(\frac{1}{2}, \frac{1}{2}\right) \geq 0$ and $\Pi_{R}^{\prime}\left(\frac{1}{2}, \frac{1}{2}\right) \leq 0$, like in a standard Dawnsian game, they do not have incentives to deviate. Suppose $L$ deviates to a platform strictly larger than $\frac{1}{2}$, then it would become the Right party, which implies that if would have incentives to converge to $\frac{1}{2}$.

\section{Proof of Proposition 1}

Proof. Lemma A.1 shows that we can constraint the analysis to the case with $x_{L} \leq \frac{1}{2} \leq x_{R}$ without loss of generality. Lemma A.2 shows strict quasiconcavity of the maximization 
problem. Hence, the first order conditions are necessary and sufficient to characterize the equilibrium. With $\mu_{L}=\mu_{R}$ equations 9 and 10 become:

$$
\begin{aligned}
\Pi_{L}^{\prime} & \equiv \frac{\partial \Pi_{L}\left(x_{L}, x_{R}\right)}{\partial x_{L}}=F(y) \frac{g(\bar{x})}{2}-f(y) G(\bar{x})+f(y) \frac{1}{2}-f(y) \eta \frac{1}{4}, \\
\Pi_{R}^{\prime} & \equiv \frac{\partial \Pi_{R}\left(x_{L}, x_{R}\right)}{\partial x_{R}}=-F(y) \frac{g(\bar{x})}{2}+f(y)[1-G(\bar{x})]-f(y) \frac{1}{2}+f(y) \eta \frac{1}{4}
\end{aligned}
$$

Remark 1 shows that convergence in the symmetric case implies $\Pi_{L}^{\prime} \geq 0$ and $\Pi_{R}^{\prime} \leq 0$. If these conditions are met, $x_{L}=\frac{1}{2}=x_{R}$ implies $y=0$. Notice that if $F(0)=0$, then there is no convergent equilibrium. When $F(0)>0$, both conditions can be re-written as $\frac{f(0)}{F(0)} \leq \frac{2}{\eta} g\left(\frac{1}{2}\right)$. Similarly, from Remark 1 we have full polarization when $\Pi_{L}^{\prime} \leq 0$ and $\Pi_{R}^{\prime} \geq 0$, evaluated at $x_{L}=0, x_{R}=1$. Using that $x_{L}=0$ and $x_{R}=1$ imply $y=1$, both conditions above can be re-written as $\frac{f(1)}{F(1)} \geq \frac{2}{\eta} g\left(\frac{1}{2}\right)$. And when there is a divergent equilibrium (without full polarization), we obtain what we call the interior/interior equilibrium when $\Pi_{L}^{\prime}=\Pi_{R}^{\prime}=0$. This equality implies that polarization is implicitly defined by $\frac{f(\bar{y})}{F(\bar{y})}=\frac{2}{\eta} g\left(\frac{1}{2}\right)$, when there is no convergent or extremism equilibrium, i.e., when the two inequalities above do not hold:

$$
\frac{f(1)}{F(1)}<\frac{2}{\eta} g\left(\frac{1}{2}\right)<\frac{f(0)}{F(0)}
$$

\section{Proof of Proposition 2}

Proof. Because of Lemma A.2 and Remark (1), solving the Lagrangian in the case $\lambda_{L}=\lambda_{R}=0$ and $\nu_{L} \geq 0 \nu_{R} \geq 0$ suffices for a convergent equilibrium. By Lemma A.1. a convergent equilibrium can only take place at $x_{L}=x_{R}=\frac{1}{2}$, which implies $\lambda_{i}=0$ for $i=L, R$. Given that $\nu_{L}$ and $\nu_{R}$ are positive, the Kuhn-Tucker conditions for a convergent equilibrium imply $\frac{\partial \Pi_{L}(x, x)}{\partial x_{L}} \geq 0$ and $\frac{\partial \Pi_{R}(x, x)}{\partial x_{R}} \leq 0$ and using that $G(0.5)=\frac{1}{2}$ they can be written for $i=L, R$ and $-i \neq i$ as:

$$
\frac{F(0)}{f(0)} g(0.5) \geq 2 \eta \frac{\mu_{L}^{\eta} \mu_{R}^{\eta}}{\left(\mu_{L}^{\eta}+\mu_{R}^{\eta}\right)^{2}}+\frac{\mu_{i}^{\eta}-\mu_{-i}^{\eta}}{\mu_{L}^{\eta}+\mu_{R}^{\eta}}
$$

Given that $\mu_{R} \geq \mu_{L}$, if the equation for $R$ is satisfied, it will also be so for $L$. Finally, note that by substituting $\mu_{L}=\mu_{R}$ we obtain the convergent condition for the proof of Proposition (1). For $F(0)=0$, there is not convergent equilibrium. 


\section{Proof of Proposition 3 and Corollary 1}

Below we provide the conditions for the existence (Proposition 3) and the characterization (Corollary 1) of the divergent equilibrium in which both parties play interior platforms. Following the classification of Corollary 1 we prove the interior/interior equilibrium in Lemma A.3 and the interior/corner equilibrium in Lemma A.4.

Lemma A.3. Let $\bar{x}^{*}=G^{-1}\left(\frac{\mu_{R}^{\eta}}{\mu_{L}^{\eta}+\mu_{R}^{\eta}}\right)$ and $y^{*}=\bar{y}$ be implicitly defined by $\frac{f(\bar{y})}{F(\bar{y})}=\frac{g\left(\bar{x}^{*}\right)}{2 \eta} \frac{\left(\mu_{L}^{\eta}+\mu_{R}^{\eta}\right)^{2}}{\mu_{L}^{\eta} \mu_{R}^{\eta}}$. Hence, there is a unique divergent interior equilibrium $x_{L}^{*}=\bar{x}^{*}-\frac{\bar{y}}{2}, x_{R}^{*}=\bar{x}^{*}+\frac{\bar{y}}{2}$ if and only if

$$
\frac{F\left(2 \bar{x}^{*}-1\right)}{f\left(2 \bar{x}^{*}-1\right)} g\left(\bar{x}^{*}\right)<2 \eta \frac{\mu_{L}^{\eta} \mu_{R}^{\eta}}{\left(\mu_{L}^{\eta}+\mu_{R}^{\eta}\right)^{2}}<\frac{F\left(2-2 \bar{x}^{*}\right)}{f\left(2-2 \bar{x}^{*}\right)} g\left(\bar{x}^{*}\right)
$$

which only holds for $\frac{\mu_{R}^{\eta}}{\mu_{R}^{\eta}+\mu_{L}^{\eta}}<G\left(\frac{3}{4}\right)$.

Proof. Because of Lemma A.2 and Remark (1), solving the Lagrangian in the case $\lambda_{i}=\nu_{i}=0$ for all $i$ suffices for a divergent interior equilibrium. Using $\bar{x}=\left(x_{L}+x_{R}\right) / 2$ and $y=x_{R}-x_{L}$, the pair $\left(x_{L}^{*}, x_{R}^{*}\right)$ is uniquely defined by the pair $\left(\bar{x}^{*}, y^{*}\right)$ with $y^{*}=\bar{y}$. We begin by proving that there is a unique $\left(\bar{x}^{*}, \bar{y}\right)$ that solves the FOCs when $\lambda_{i}=0=\nu_{i}$ for all $i$. From $\frac{\partial \mathcal{L}_{L}}{\partial x_{L}}+\frac{\partial \mathcal{L}_{R}}{\partial x_{R}}=0$ and $G(x)$ strictly increasing in $x$, we obtain the unique $\bar{x}^{*}$ that solves the FOCs:

$$
G\left(\bar{x}^{*}\right)=\frac{\mu_{R}^{\eta}}{\mu_{L}^{\eta}+\mu_{R}^{\eta}} \Longleftrightarrow \bar{x}^{*}=G^{-1}\left(\frac{\mu_{R}^{\eta}}{\mu_{L}^{\eta}+\mu_{R}^{\eta}}\right) .
$$

Plugging $\bar{x}^{*}$ in 7 or 8 , we obtain $\frac{f(\bar{y})}{F(\bar{y})}=\frac{g\left(\bar{x}^{*}\right)}{2 \eta} \frac{\left(\mu_{L}^{\eta}+\mu_{R}^{\eta}\right)^{2}}{\mu_{L}^{\eta} \mu_{R}^{\eta}}$. Since the conversion rate is increasing and $g\left(\bar{x}^{*}\right)$ can be treated as a constant, there is a unique polarization level $\bar{y}$ that solves the FOCs. Hence, we obtain the unique

$$
x_{L}^{*}=\bar{x}^{*}-\frac{\bar{y}}{2}
$$

and

$$
x_{R}^{*}=\bar{x}^{*}+\frac{\bar{y}}{2}
$$

that solve the FOCs. Note that for $\mu_{L}=\mu_{R}$ we immediately obtain that $G\left(\bar{x}^{*}\right)=\frac{1}{2}$ and by symmetry o $G(x)$ that $\bar{x}^{*}=\frac{1}{2}$

Finally, we have to check that the above solutions, $x_{L}^{*}$ and $x_{R}^{*}$ lie within the corresponding policy sub-space $x_{L}^{*} \in\left(0, \frac{1}{2}\right)$ and $x_{R}^{*} \in\left(\frac{1}{2}, 1\right)$. First, $G(x)$ is increasing, so its inverse is as well. Hence, from $x_{L}^{*} \in\left(0, \frac{1}{2}\right)$, we obtain that $\bar{y}>2 G^{-1}\left(\frac{\mu_{R}^{\eta}}{\mu_{R}^{\eta}+\mu_{L}^{\eta}}\right)-1$, and 
from $x_{R}^{*} \in\left(\frac{1}{2}, 1\right)$, we obtain that $\bar{y}<2-2 G^{-1}\left(\frac{\mu_{R}^{\eta}}{\mu_{R}^{\eta}+\mu_{L}^{\eta}}\right)$. Hence, it must be the case that

$$
\bar{y} \in\left(2 G^{-1}\left(\frac{\mu_{R}^{\eta}}{\mu_{R}^{\eta}+\mu_{L}^{\eta}}\right)-1,2-2 G^{-1}\left(\frac{\mu_{R}^{\eta}}{\mu_{R}^{\eta}+\mu_{L}^{\eta}}\right)\right) \Longleftrightarrow \bar{y} \in\left(2 \bar{x}^{*}-1,2-2 \bar{x}^{*}\right),
$$

which only holds if $\frac{\mu_{R}^{\eta}}{\mu_{R}^{\eta}+\mu_{L}^{\eta}}<G\left(\frac{3}{4}\right)$. Also, in Equation 14, we can use the definition of $\bar{y}$ and that the rate $\frac{F(x)}{f(x)}$ is increasing and invertible to obtain the conditions of the equilibrium in terms of the conversion rate:

$$
\begin{gathered}
\frac{F\left(2 G^{-1}\left(\frac{\mu_{R}^{\eta}}{\mu_{R}^{\eta}+\mu_{L}^{\eta}}\right)-1\right)}{f\left(2 G^{-1}\left(\frac{\mu_{R}^{\eta}}{\mu_{R}^{\eta}+\mu_{L}^{\eta}}\right)-1\right)}<\frac{F(\bar{y})}{f(\bar{y})} \frac{1}{g\left(G^{-1}\left(\frac{\mu_{R}^{\eta}}{\mu_{R}^{\eta}+\mu_{L}^{\eta}}\right)\right)}<\frac{F\left(2-2 G^{-1}\left(\frac{\mu_{R}^{\eta}}{\mu_{R}^{\eta}+\mu_{L}^{\eta}}\right)\right)}{f\left(2-2 G^{-1}\left(\frac{\mu_{R}^{\eta}}{\mu_{R}^{\eta}+\mu_{L}^{\eta}}\right)\right)} \\
\Longleftrightarrow \\
\frac{F\left(2 \bar{x}^{*}-1\right)}{f\left(2 \bar{x}^{*}-1\right)}<\frac{F(\bar{y})}{f(\bar{y})} \frac{1}{g\left(\bar{x}^{*}\right)}<\frac{F\left(2-2 \bar{x}^{*}\right)}{f\left(2-2 \bar{x}^{*}\right)}
\end{gathered}
$$

Note that for the symmetric cost case, $\mu_{L}=\mu_{R}$, the above simplifies to $\frac{F(0)}{f(0)} g\left(\frac{1}{2}\right)<$ $\frac{\eta}{2}<\frac{F(1)}{f(1)} g\left(\frac{1}{2}\right)$

Lemma A.4. $\left(0<x_{L}^{*} \leq \frac{1}{2}, x_{R}^{*}=1\right)$ Let $\bar{x}^{*}=G^{-1}\left(\frac{\mu_{R}^{\eta}}{\mu_{L}^{\eta}+\mu_{R}^{\eta}}\right)$ and $\bar{y}$ be implicitly defined by $\frac{f(\bar{y})}{F(\bar{y})}=\frac{g\left(\bar{x}^{*}\right)}{2 \eta} \frac{\left(\mu_{L}^{\eta}+\mu_{R}^{\eta}\right)^{2}}{\mu_{L}^{\eta} \mu_{R}^{\eta}}$. Let $x_{L}^{*}$ be implicitly defined by $2 G\left(\frac{1+x_{L}^{*}}{2}\right)=\frac{F\left(1-x_{L}^{*}\right)}{f\left(1-x_{L}^{*}\right)} g\left(\frac{1+x_{L}^{*}}{2}\right)+$ $2 \frac{\mu_{R}^{\eta}}{\mu_{R}^{\eta}+\mu_{L}^{\eta}}-2 \eta \frac{\mu_{R}^{\eta} \mu_{L}^{\eta}}{\left(\mu_{R}^{\eta}+\mu_{L}^{\eta}\right)^{2}}$.

For $\frac{\mu_{R}^{\eta}}{\mu_{R}^{\eta}+\mu_{L}^{\eta}}<G\left(\frac{3}{4}\right)$, there is a unique equilibrium $\left(x_{L}^{*}, 1\right)$ if and only if

$$
\frac{F\left(2-2 \bar{x}^{*}\right)}{f\left(2-2 \bar{x}^{*}\right)} g\left(\bar{x}^{*}\right)<2 \eta \frac{\mu_{L}^{\eta} \mu_{R}^{\eta}}{\left(\mu_{L}^{\eta}+\mu_{R}^{\eta}\right)^{2}}<\frac{F(1)}{f(1)} g(0.5)+\frac{\mu_{R}^{\eta}-\mu_{L}^{\eta}}{\mu_{R}^{\eta}+\mu_{L}^{\eta}}
$$

For $\frac{\mu_{R}^{\eta}}{\mu_{R}^{\eta}+\mu_{L}^{\eta}} \geq G\left(\frac{3}{4}\right)$, there is a unique equilibrium $\left(x_{L}^{*}, 1\right)$ if and only if

$$
\frac{F\left(\frac{1}{2}\right)}{f\left(\frac{1}{2}\right)} g\left(\frac{3}{4}\right)-2 G\left(\frac{3}{4}\right)+\frac{2 \mu_{R}^{\eta}}{\mu_{R}^{\eta}+\mu_{L}^{\eta}}<2 \eta \frac{\mu_{L}^{\eta} \mu_{R}^{\eta}}{\left(\mu_{L}^{\eta}+\mu_{R}^{\eta}\right)^{2}}
$$

Proof. Because of Lemma A.2 and Remark (1), solving the Lagrangian in the case where $\nu_{L}=\nu_{R}=\lambda_{L}=0$ and $\lambda_{R} \geq 0$ is sufficient to find an equilibrium where $x_{L}^{*} \leq \frac{1}{2}$ and $x_{R}^{*}=1$. Let $\bar{x}=\frac{1+x_{L}}{2}$ and $y=1-x_{L}$, then taking into account the conditions on 
the Lagrange-multipliers:

$$
\begin{aligned}
\frac{\partial \prod_{L}\left(x_{L}, x_{R}=1\right)}{\partial x_{L}}+\frac{\partial \prod_{R}\left(x_{L}, x_{R}=1\right)}{\partial x_{R}}=\lambda_{R} & \geq 0 \\
\frac{\mu_{R}^{\eta}}{\mu_{R}^{\eta}+\mu_{L}^{\eta}} & \geq G\left(\frac{x_{L}+1}{2}\right) \\
2 G^{-1}\left(\frac{\mu_{R}^{\eta}}{\mu_{R}^{\eta}+\mu_{L}^{\eta}}\right) & \geq x_{L}+1
\end{aligned}
$$

Lemma A.1 and Equation 15 imply that $x_{L} \leq \min \left\{2 G^{-1}\left(\frac{\mu_{R}^{\eta}}{\mu_{R}^{\eta}+\mu_{L}^{\eta}}\right)-1, \frac{1}{2}\right\}$. Taking into account that $2 G^{-1}\left(\frac{\mu_{R}^{\eta}}{\mu_{R}^{\eta}+\mu_{L}^{\eta}}\right)-1<\frac{1}{2} \Longleftrightarrow \frac{\mu_{R}^{\eta}}{\mu_{R}^{\eta}+\mu_{L}^{\eta}}<G\left(\frac{3}{4}\right)$, we solve the following:

- If $\frac{\mu_{R}^{\eta}}{\mu_{R}^{\eta}+\mu_{L}^{\eta}}<G\left(\frac{3}{4}\right)$, then $\left.\frac{\partial \Pi_{L}\left(x_{L}, 1\right)}{\partial x_{L}}\right|_{x_{L}=2 \bar{x}^{*}-1} \leq 0$ must hold in equilibrium. Plugging in $x_{L}=2 \bar{x}^{*}-1$ and $x_{R}=1$ and using $G\left(\bar{x}^{*}\right)=\frac{\mu_{R}^{\eta}}{\mu_{R}^{\eta}+\mu_{L}^{\eta}}$ in Equation (7) we obtain

$$
\frac{F\left(2-2 \bar{x}^{*}\right)}{f\left(2-2 \bar{x}^{*}\right)} g\left(\bar{x}^{*}\right) \leq 2 \eta \frac{\mu_{L}^{\eta} \mu_{R}^{\eta}}{\left(\mu_{L}^{\eta}+\mu_{R}^{\eta}\right)^{2}}
$$

- If $\frac{\mu_{R}^{\eta}}{\mu_{R}^{\eta}+\mu_{L}^{\eta}} \geq G\left(\frac{3}{4}\right)$, then $\left.\frac{\partial \Pi_{L}\left(x_{L}, 1\right)}{\partial x_{L}}\right|_{x_{L}=0.5} \leq 0$ must hold in equilibrium. Using $x_{L}=0.5$ in Equation (7) we obtain

$$
\begin{array}{r}
\frac{F(0.5)}{f(0.5)}-\frac{2}{g\left(\frac{3}{4}\right)} G\left(\frac{3}{4}\right)+\frac{2}{g\left(\frac{3}{4}\right)} \frac{\mu_{R}^{\eta}}{\mu_{R}^{\eta}+\mu_{L}^{\eta}}-\frac{2}{g\left(\frac{3}{4}\right)} \eta \frac{\mu_{R}^{\eta} \mu_{L}^{\eta}}{\left(\mu_{R}^{\eta}+\mu_{L}^{\eta}\right)^{2}} \\
\leq 0 \\
\frac{F(0.5)}{f(0.5)} g\left(\frac{3}{4}\right)-2 G\left(\frac{3}{4}\right)+\frac{2 \mu_{R}^{\eta}}{\mu_{R}^{\eta}+\mu_{L}^{\eta}} \leq 2 \eta \frac{\mu_{L}^{\eta} \mu_{R}^{\eta}}{\left(\mu_{L}^{\eta}+\mu_{R}^{\eta}\right)^{2}}
\end{array}
$$

Finally, the fully divergent equilibrium is excluded when we assure $x_{L}>0$, i.e., when $2 \eta \frac{\mu_{L}^{\eta} \mu_{R}^{\eta}}{\left(\mu_{L}^{\eta}+\mu_{R}^{\eta}\right)^{2}}<\frac{F(1)}{f(1)} g(0.5)+\frac{\mu_{R}^{\eta}-\mu_{L}^{\eta}}{\mu_{R}^{\eta}+\mu_{L}^{\eta}}$ (see Proposition 4 below). Notice that when $\frac{\mu_{R}^{\eta}}{\mu_{R}^{\eta}+\mu_{L}^{\eta}} \geq G\left(\frac{3}{4}\right)$, the latter inequality always holds 14

\section{Proof of Proposition 4}

Proof. Because of Lemma A.2 and Remark (1), solving the Lagrangian in the case where $\nu_{i}=0$ and $\lambda_{i} \geq 0$ for all $i$ is sufficient to find an equilibrium with $x_{L}=0$ and

${ }^{14}$ Recall that $\eta \leq \bar{\eta}$ implies that $2 \eta \frac{\mu_{L}^{\eta} \mu_{R}^{\eta}}{\left(\mu_{L}^{\eta}+\mu_{R}^{\eta}\right)^{2}}<2 \frac{\mu_{L}^{\eta}+\mu_{R}^{\eta}}{\mu_{R}^{\eta}} \frac{\mu_{L}^{\eta} \mu_{R}^{\eta}}{\left(\mu_{L}^{n}+\mu_{R}^{\eta}\right)^{2}}=2 \frac{\mu_{L}^{\eta}}{\mu_{L}^{\eta}+\mu_{R}^{\eta}}$. Also $2 \frac{\mu_{L}^{\eta}}{\mu_{L}^{\eta}+\mu_{R}^{\eta}} \leq$ $\frac{\mu_{R}^{\eta}-\mu_{L}^{\eta}}{\mu_{R}^{\eta}+\mu_{L}^{\eta}} \Longleftrightarrow \mu_{R}^{\eta} \geq 3 \mu_{L}^{\eta}$. Then, single-peakness and symmetry of $g(x)$ imply $G\left(\frac{3}{4}\right)>\frac{3}{4}$. Finally $\mu_{R}^{\eta} \geq 3 \mu_{L}^{\eta}$ follows directly from $\frac{\mu_{R}^{\eta}}{\mu_{R}^{\eta}+\mu_{L}^{\eta}} \geq G\left(\frac{3}{4}\right)$ 
$x_{R}=1$. Hence, from Equation 7 and 8 ,

$$
\lambda_{L} \geq 0 \text { iff } \frac{F(1)}{f(1)} g(0.5)+\frac{\mu_{-i}^{\eta}-\mu_{i}^{\eta}}{\mu_{L}^{\eta}+\mu_{R}^{\eta}} \leq 2 \eta \frac{\mu_{L}^{\eta} \mu_{R}^{\eta}}{\left(\mu_{L}^{\eta}+\mu_{R}^{\eta}\right)^{2}} .
$$

As $\mu_{R} \geq \mu_{L}$, the condition for party $L$ is the sufficient one. Last, $\frac{F(1)}{f(1)} g(0.5)>0$, so a necessary condition for a fully divergent equilibrium is

$$
2 \eta \mu_{L}^{\eta} \mu_{R}^{\eta} \geq \mu_{R}^{2 \eta}-\mu_{L}^{2 \eta}=\left(\mu_{R}^{\eta}-\mu_{L}^{\eta}\right)\left(\mu_{R}^{\eta}+\mu_{L}^{\eta}\right) \geq\left(\mu_{R}^{\eta}-\mu_{L}^{\eta}\right) \eta \mu_{R}^{\eta}
$$

where the last inequality follows from $\eta \leq \bar{\eta}$. And the expression above simplifies to $3 \mu_{L}^{\eta} \geq \mu_{R}^{\eta}$

\subsection{Comparative statics}

\section{Proof of Lemma (2).}

Proof. By Proposition (1), the interior equilibrium arises if $\eta \in\left[2 g\left(\frac{1}{2}\right) \frac{F(0)}{f(0)}, 2 g\left(\frac{1}{2}\right) \frac{F(1)}{f(1)}\right]$.

Using the implicit function theorem we can write: $\frac{\partial \bar{y}}{\partial \eta}=\frac{1}{2 g(0.5)\left[\frac{F(\bar{y})}{f(\bar{y})}\right]^{\prime}}$. Then,

$$
\frac{\partial \mu e_{i}^{*}\left(x_{L}^{*}, x_{R}^{*}\right)}{\partial \eta}=-f(\bar{y}) \frac{\partial \bar{y}}{\partial \eta} \frac{\eta}{4}+\frac{1-F(\bar{y})}{4}=-\frac{f(\bar{y})}{2 g(0.5)\left[\frac{F(\bar{y})}{f(\bar{y})}\right]^{\prime}} \frac{\eta}{4}+\frac{1-F(\bar{y})}{4} .
$$

Hence,

$$
\frac{\partial \mu e_{i}^{*}\left(x_{L}^{*}, x_{R}^{*}\right)}{\partial \eta} \geq 0 \Longleftrightarrow 1-F(\bar{y}) \geq \frac{f(\bar{y})}{2 g(0.5)\left[\frac{F(\bar{y})}{f(\bar{y})}\right]^{\prime}} \eta \Longleftrightarrow \eta \leq \frac{1-F(\bar{y})}{f(\bar{y})} 2 g(0.5)\left[\frac{F(\bar{y})}{f(\bar{y})}\right]^{\prime}
$$

where $\left[\frac{F(\bar{y})}{f(\bar{y})}\right]^{\prime}$ is a positive number by log-concavity of $F(y)$.

\section{Proof of Lemma (3)}

Proof. By Proposition [1], the interior equilibrium arises if $\eta \in\left[2 g\left(\frac{1}{2}\right) \frac{F(0)}{f(0)}, 2 g\left(\frac{1}{2}\right) \frac{F(1)}{f(1)}\right]$. Then,

$$
\frac{\partial \mu e_{i}^{*}\left(x_{L}^{*}, x_{R}^{*}\right)}{\partial \rho}=-\frac{\partial F\left(y^{*}\right)}{\partial \rho} \frac{\eta}{4}-\frac{\partial F\left(y^{*}\right)}{\partial y^{*}} \frac{\partial y^{*}}{\partial \rho} \frac{\eta}{4}
$$

and hence $\frac{\partial \mu e_{i}^{*}\left(x_{L}^{*}, x_{R}^{*}\right)}{\partial \rho} \geq 0$ if and only if $-\frac{\partial F(\bar{y})}{\partial \rho} \geq f(\bar{y}) \frac{\partial \bar{y}}{\partial \rho}$. 


\subsection{Voters' behavior}

In this section we formalize and derive two main assumptions of the model. Namely, a) the endogenous division of voters across ideological and impressionable (via semiorder lexicographic preferences or a model of salience), and b) the impressionable voters' behavior that results in a Tullock contest during the campaign stage.

In their "strict" formulation, lexicographic preferences require a tie in the first attribute to compare alternatives over a second attribute. In their "weak" formulation (or semiorder, (Tversky, 1969)), small differences between alternatives in the first attribute also lead to indifference in that attribute. In other words, small differences on the first attribute are disregarded (Fishburn, 1974). This is the exact intuition we presented in our model where as polarization decreases and platforms look more alike, individuals turn their attention to campaigns instead of platforms. In particular, semiorder preferences with binary choices (i.e., two platforms in our setup) are unrestrictive in the sense of Manzini and Mariotti (2012), and the use of semiorder lexicographic preferences microfounds the aggregate behavior presented in the main text.

Assume a population of measure one, in which voters have semiorder lexicographic preferences and are heterogeneous in two dimensions. First, they draw an ideal policy $x \in[0,1]$ from $G(x)$. Second, voters also draw a level of sensitivity, $\phi$, towards differences in the ideology space, from $F_{\phi}(y)=\operatorname{Pr}(\phi<y)$. Hence, $\phi$ is the minimal distance between the two platforms that a voter considers to be "relevant" or "distinguishable" ${ }^{15}$ When the distance between two platforms is less than $\phi$ the voter treats them as identical and moves to the second attribute, electoral advertising, $e_{i}$. Although $x$ is ex-post irrelevant for impressionable voters, all individuals are identified by the pair $(x, \phi)$.

The above features can be represented in an analytical manner by adapting the semiorder lexicographic structure proposed by Luce (1978). Considering voter $(x, \phi)$, we can write the voter's evaluation of party $i$ as:

$$
\vartheta_{x, \phi}(i)=-\left|x-x_{i}\right| \Upsilon\left(\phi \leq x_{R}-x_{L}\right)+t^{i}\left(e_{R}, e_{L}, \theta^{i}\right)\left[1-\Upsilon\left(\phi \leq x_{R}-x_{L}\right)\right]
$$

where $\Upsilon\left(\phi \leq x_{R}-x_{L}\right)$ is an indicator function taking value 1 when a voter is ideological, i.e., $\phi \leq x_{R}-x_{L}=y$, and 0 when a voter is impressionable, i.e., $\phi>x_{R}-x_{L}=y$, as in the main text. Without loss of generality $-\left|x-x_{i}\right|$ is the utility derived from voting for party $i \in\{L, R\}$ according to ideology; and $t^{i}\left(e_{R}, e_{L}, \theta^{i}\right)$ is the impressionable voter's utility from voting for party $i \in\{L, R\}$ according to advertisements.

Let $\theta^{i}$ in $t^{i}\left(e_{R}, e_{L}, \theta^{i}\right)$ be a random variable (from the candidates' points of view) that

\footnotetext{
${ }^{15}$ In terms of the experimental literature in human perception (or psychophysics), $\phi$ can be interpreted as the just-noticeable difference.
} 
captures how much of a party's advertisement "leaks" to voters in the following way $t^{i}\left(e_{R}, e_{L}, \theta^{i}\right)=\log \left(e_{i}^{\eta}\right)+\theta^{i}$. Similarly to multinomial applications in industrial organization (Nevo, 2000) and political economy (Casas, Fawaz, and Trindade, 2016), assume $\theta^{i}$ to be drawn i.i.d. from an type $I$ extreme-value distribution. As in McFadden (1974), the probability $\operatorname{Pr}\left(t^{L}>t^{R}\right)$ - which in our case is the probability that an impressionable voter votes for $L$ - becomes the contest success function $\operatorname{Pr}\left(t^{L}>t^{R}\right)=\frac{e_{L}^{\eta}}{e_{L}^{\eta}+e_{R}^{\eta}}$. With a continuum of voters, we interpret this probability as the share of impressionable voters that vote for $L$. On the same lines, (Jia, 2008, Jia, Skaperdas, and Vaidya, 2013) show that for $t^{i}\left(e_{R}, e_{L}, \theta^{i}\right)=e_{i} \theta^{i}$, if $\theta^{L}$ and $\theta^{R}$ follow independent inverse exponential distributions with parameter $\eta>0$, the probability also becomes $\operatorname{Pr}\left(t^{L}>t^{R}\right)=\frac{e_{L}^{\eta}}{e_{L}^{\eta}+e_{R}^{\eta}}, 16$

For simplicity, let $F_{\phi}(y)$ be written as $F(y)$, which can be used to denote the proportion of ideological voters given a level of polarization $y$. Platform preferences and ideology sensitivity are assumed to be independent. Consequently, for a given pair of policy platforms $x_{L}$ and $x_{R}$, the votes of ideological and impressionable voters can be independently aggregated (integrating over $\phi$ ). By integrating over $\theta_{L}$ and $\theta_{R}$ under the above distributional assumptions, the impressionable vote share of party $i$ is $S_{I m p}^{i}=\frac{e_{i}^{\eta}}{e_{L}^{\eta}+e_{R}^{\eta}}$. For ideologogical voters, by integraing over $x$ one immediately obtains that for party $L$, $S_{\text {Idl }}^{L}=G(\bar{x})$ if $x_{L} \neq x_{R}$ and $S_{I d l}^{L}=\frac{1}{2}$ if $x_{L}=x_{R}$. By taking into account the above, parties' vote shares can be immediately written as in Equation (1).

\section{Salience and attention}

Our model can also be interpreted as an extreme case of (Bordalo et al. 2012, 2013a) b, 2015), in which "salient thinkers" give more weight to attributes that exhibit greater heterogeneity in the available choice set.

In the papers on salience and attention, the salient attribute is the one in which the differences are more pronounced. The less salient attribute receives less weight. For instance, in Bordalo et al. (2015) they look at price and quality: if quality is the salient attribute, the utility from consuming good $k$ is $q_{k}-\omega p_{k}$ with $\omega$ exogenously determined and in $[0,1]$. Instead of comparing attributes, we compare the platforms differentiation with a baseline level of polarization, $\phi$, which is exogenously drawn from $F_{\phi}(y)$. Thus, for $\phi$ smaller than the equilibrium polarization, voters take platforms as the salient attribute. In particular, for $\phi \leq y, \omega=0$. And for $\phi \geq y, 1-\omega=0$. Thus, one can consider the rank-based weighting salience function proposed by Bordalo et al. 2012, 2013b, 2015, where:

$$
\vartheta_{x, \phi}(i)=-\left(\left|x-x_{i}\right|\right) \omega+t^{i}\left(e_{L}, e_{R}, \theta^{i}\right)(1-\omega)
$$

The heterogeneity of "salient thinkers" is given by $F_{\phi}(y)$, and everything else is exactly

\footnotetext{
${ }^{16}$ The assumption that party shocks are identical to all individuals is made without loss of generality, as long as they are independent of ideology.
} 
as in the previous section. 


\section{References}

Alcalde, J. And M. DAhm (2007): "Tullock and Hirshleifer: a meeting of the minds," Review of Economic Design, 11, 101-124.

(2010): "Rent seeking and rent dissipation: a neutrality result," Journal of Public Economics, 94, 1-7.

Amegashie, J. A. (2006): "A contest success function with a tractable noise parameter," Public Choice, 126, 135-144.

Amorós, P. AND M. S. PUY (2013): "Issue convergence or issue divergence in a political campaign?" Public Choice, 155, 355-371.

Aragonés, E., M. Castanheira, And M. Giani (2015): "Electoral Competition through Issue Selection," American Journal of Political Science, 59, 71-90.

Aragonès, E. AND D. Xefteris (2017): "Imperfectly informed voters and strategic extremism," International Economic Review, 58, 439-471.

Ashworth, S. And E. Bueno De Mesquita (2009): "Elections with platform and valence competition," Games and Economic Behavior, 67, 191-216.

BAIK, K. H. (1994): "Effort levels in contests with two asymmetric players," Southern Economic Journal, 367-378.

Balart, P., S. M. Chowdhury, and O. Troumpounis (2017): "Linking individual and collective contests through noise level and sharing rules," Economics Letters, 155, $126-130$.

BARon, D. P. (1994): "Electoral competition with informed and uninformed voters." American Political Science Review, 88, 33-47.

Beviá, C. And L. C. Corchón (2015): "Relative difference contest success function," Theory and Decision, 78, 377-398.

Bordalo, P., N. Gennaioli, And A. Shleifer (2012): "Salience theory of choice under risk," The Quarterly journal of economics, 127, 1243-1285.

(2013a): "Salience and asset prices," The American Economic Review, 103, 623628.

(2013b): "Salience and consumer choice," Journal of Political Economy, 121, 803-843. 
(2015): "Competition for attention," The Review of Economic Studies, 83, 481513.

Bushong, B., M. Rabin, And J. Schwartzstein (2017): "A model of relative thinking," HBS Working Paper.

Callander, S. And C. H. Wilson (2006): "Context-dependent Voting," Quarterly Journal of Political Science, 1, 227-254.

Cardona, D., J. De Freitas, and A. Rubí-Barceló (2018): "Polarization or Moderation? Intra-group heterogeneity in endogenous-policy contests," Tech. rep., Universitat de les Illes Balears, Departament d'Economía Aplicada.

Carrillo, J. D. and M. Castanheira (2008): "Information and strategic political polarisation," The Economic Journal, 118, 845-874.

Casas, A., Y. Fawaz, And A. Trindade (2016): "Surprise me if you can: The influence of newspaper endorsements in u.s. presidential elections," Economic Inquiry.

Corchón, L. C. (2007): "The theory of contests: a survey," Review of Economic Design, $11,69-100$.

DENTER, P. (2017): "Campaign contests," REPEC working paper.

Downs, A. (1957): An Economic Theory of Democracy, Harper, New York.

EguiA, J. X. And A. Nicolò (2018): "Information and targeted spending," Theoretical Economics, forthcoming.

Epstein, G. S. AND S. Nitzan (2004): "Strategic restraint in contests," European Economic Review, 48, 201-210.

Ewerhart, C. (2015): "Mixed equilibria in Tullock contests," Economic Theory, 60, 59-71.

- (2017): "Revenue ranking of optimally biased contests: The case of two players," Economics Letters, 157, 167-170.

Falmagne, J.-C. (2002): Elements of psychophysical theory, 6, Oxford University Press on Demand.

Fishburn, P. C. (1974): "Exceptional paper-lexicographic orders, utilities and decision rules: A survey," Management Science, 20, 1442-1471. 
Grossman, G. M. And E. Helpman (1996): "Electoral competition and special interest politics," The Review of Economic Studies, 63, 265-286.

Herrera, H., D. K. Levine, and C. Martinelli (2008): "Policy platforms, campaign spending and voter participation," Journal of Public Economics, 92, 501-513.

IARYCZOWER, M. AND A. MATtozzI (2013): "On the nature of competition in alternative electoral systems," The Journal of Politics, 75, 743-756.

JIA, H. (2008): "A stochastic derivation of the ratio form of contest success functions," Public Choice, 135, 125-130.

Jia, H., S. Skaperdas, And S. VAidya (2013): "Contest functions: Theoretical foundations and issues in estimation," International Journal of Industrial Organization, 31, $211-222$.

Konrad, K. A. (2009): Strategy and Dynamics in Contests, Oxford UK: Oxford University Press.

Köszegi, B. And A. Szeidl (2012): "A model of focusing in economic choice," The Quarterly journal of economics, 128, 53-104.

LELAND, J. W. (1994): "Generalized similarity judgments: An alternative explanation for choice anomalies," Journal of Risk and Uncertainty, 9, 151-172.

Luce, R. D. (1956): "Semiorders and a theory of utility discrimination," Econometrica, Journal of the Econometric Society, 178-191.

Manzini, P. And M. Mariotti (2012): "Choice by lexicographic semiorders," Theoretical Economics, 7, 1-23.

MCFAdDen, D. (1974): "The measurement of urban travel demand," Journal of Public Economics, 3, 303 - 328.

Meirowitz, A. (2008): "Electoral contests, incumbency advantages, and campaign finance," The Journal of Politics, 70, 681-699.

NBC (2017): "How Big Data Broke American Politics," NBC news by Chuck Todd and Carrie Dann (story adapted from a presentation NBC News' Chuck Todd delivered at SXSW in Austin, Texas), published on March $14^{\text {th }} 201 \%$.

Nevo, A. (2000): "A Practitioner's Guide to Estimation of Random-Coefficients Logit Models of Demand," Journal of Economics \& Management Strategy, 9, 513-548. 
Newton, K. (1999): "Mass media effects: mobilization or media malaise?" British Journal of Political Science, 29, 577-599.

Nickerson, D. W. And T. Rogers (2014): "Political campaigns and big data," Journal of Economic Perspectives, 28, 51-74.

Nitzan, S. (1994): "Modelling rent-seeking contests," European Journal of Political Economy, 10, 41-60.

Norris, P. (2000): A virtuous circle: Political communications in postindustrial societies, Cambridge University Press.

Nti, K. O. (1999): "Rent-seeking with asymmetric valuations," Public Choice, 98, 415430.

Nunnari, S. And J. ZÁpal (2017): "A Model of Focusing in Political Choice," CEPR Discussion Paper No. $1240 \%$.

Pastine, I. And T. PAstine (2012): "Incumbency advantage and political campaign spending limits," Journal of Public Economics, 96, 20-32.

Poole, K. T. And H. Rosenthal (1984): "The polarization of American politics," The Journal of Politics, 46, 1061-1079.

Prummer, A. (2018): "Spatial Advertisement in Political Campaigns," mimeo.

Rivas, J. (2017): "Lobbying, Campaign Contributions and Political Competition," Bath Economics Research Working Paper.

Rubinstein, A. (1988): "Similarity and decision-making under risk (Is there a utility theory resolution to the Allais paradox?)," Journal of economic theory, 46, 145-153.

SABATO, L. (1981): The rise of political consultants: New ways of winning elections, vol. 5114, Basic Books (AZ).

Serena, M. And L. Corchón (2018): "Contests theory: a survey," in Handbook of game theory and industrial organization, ed. by L. Corchón and M. A. Marini, Edward Elgar, forthcoming.

SkAPERDAS, S. (1996): "Contest success functions," Economic theory, 7, 283-290.

Spiegler, R. (2014): "Competitive framing," American Economic Journal: Microeconomics, 6, 35-58. 
TiRole, J. (1988): The Theory of Industrial Organization, MIT Press.

Tullock, G. (1980): "Efficient Rent Seeking," in Toward a Theory of the Rent-Seeking Society, ed. by J. M. Buchanan, R. D. Tollison, and G. Tullock, College Station, TX: Texas A\&M University Press, 97-112.

Tversky, A. (1969): "Intransitivity of Preferences," Psychological Review, 76, 31-48.

WANG, Z. (2010): "The optimal accuracy level in asymmetric contests," The BE Journal of Theoretical Economics, 10.

Zakharov, A. V. (2009): "A model of candidate location with endogenous valence," Public Choice, 138, 347-366. 